\title{
Self-consistent nonspherical isothermal halos embedding zero-thickness disks ${ }^{\star}$
}

\author{
N. C. Amorisco ${ }^{1}$ and G. Bertin ${ }^{2}$ \\ 1 Institute of Astronomy, Madingley Road, Cambridge CB3 OHA, UK \\ e-mail: amorisco@ast.cam.ac.uk \\ 2 Dipartimento di Fisica, Università degli Studi di Milano, via Celoria 16, 20133 Milano, Italy \\ e-mail: giuseppe. bertin@unimi.it
}

Received 9 March 2010 / Accepted 8 May 2010

\begin{abstract}
Context. That the rotation curves of spiral galaxies are generally flat in the outer regions is commonly considered an indication that galaxy disks are embedded in quasi-isothermal halos. In practice, disk-halo decompositions of galaxy rotation curves are performed in a parametric way by modeling the halo force contribution by means of expressions that approximately describe the properties of the regular isothermal sphere or other spherical density distributions suggested by cosmological simulations.

Aims. In this paper we construct self-consistent models of nonspherical isothermal halos embedding a zero-thickness disk, by assuming that the halo distribution function is a Maxwellian. The general method developed here can also be used to study the properties of other physically-based choices for the halo distribution function and to the case of a disk accompanied by a bulge.

Methods. The construction was performed by means of an iterative procedure that generalizes a method introduced in the past to construct spheroidal models of rotating elliptical galaxies. In a preliminary investigation, which set the empirical framework to study the self-consistent models developed in this paper, we note the existence of a fine tuning between the scalelengths $R_{\Omega}$ and $h$, respectively characterizing the rise of the rotation curve and the luminosity profile of the disk, which surprisingly applies to both high surface brightness and low surface brightness galaxies in similar ways. We show that this empirical correlation identifies a much stronger conspiracy than the one required by the smoothness and flatness of the rotation curve and often referred to as disk-halo conspiracy. Results. As a natural property, the self-consistent models presented in this paper are found to be characterized by smooth and flat rotation curves for very different disk-to-halo mass ratios, hence suggesting that conspiracy is not as dramatic as often imagined. For a typical, observed rotation curve, with asymptotically flat rotation curve at $V_{\infty}$ (the precise value of which can also be treated as a free parameter), and a typical density profile of the disk, the relevant self-consistent models are characterized by two dimensionless parameters, which correspond to the dimensional scales (the disk mass-to-light ratio $M / L$ and the halo central density) of standard disk-halo decompositions. We find that, if the rotation curve is decomposed by means of our self-consistent models, the disk-halo degeneracy is removed and that typical rotation curves are fitted by models that are below the maximum-disk prescription. Similar results are obtained from a study of NGC 3198. Finally, we quantify the flattening of the spheroidal halo, which is significant, especially on the scale of the visible disk.
\end{abstract}

Key words. galaxies: spiral - galaxies: structure - galaxies: halos - galaxies: kinematics and dynamics galaxies: individual: NGC 3198

\section{Introduction}

The study of the rotation curve of spiral galaxies (from the case of M31, van de Hulst et al. 1957, to the systematic investigation of nearby galaxies in the 70s) has led to convincing evidence for the presence of dark matter halos (e.g., see Roberts 1976). To this discovery, HI rotation curves, which often sample regions well beyond the bright optical disk, played a decisive role (van Albada et al. 1985; van Albada \& Sancisi 1986).

In this context, it has been suggested that the existence of a halo of dark matter introduces a problem of fine tuning or "conspiracy" (van Albada \& Sancisi 1986). The coexisting dark and visible components, while expected to have different formation and evolution histories (even if naturally coupled by their mutual gravitational interaction), must always cooperate to produce a gravitational field inversely proportional to the radius, so that the resulting rotation curve remains flat from the inner regions (generally thought to be dominated by the visible matter) out to large

* Appendices $\mathrm{A}$ and $\mathrm{B}$ are only available in electronic form at http://www . aanda. org radii (where the field is dominated by the diffuse dark halo). This puzzling feature is accompanied by a serious problem of degeneracy. In fact, from a detailed study of the so-called diskhalo decomposition, it has been shown that, in general, the available photometric profile and the corresponding velocity datapoints can be modeled in many different ways, either by heavy or by light disks, through an ill-determined mass-to-light ratio $M / L$ for the disk. In general, the available data are only able to set an upper limit to such $M / L$, identifying the so-called maximumdisk solution, for the model that maximizes the contribution of visible matter. Typically, the maximum-disk solution is able to fit the rotation curve out to the optical radius (Kalnajs 1983; Kent 1986).

It was immediately realized that the flatness of the outer rotation curves suggests that the dark halo embedding the visible disk might have a structure similar to that of the classical isothermal sphere, because outside the central core such a sphere is characterized by a density distribution declining approximately as $\rho \sim 1 / r^{2}$. Curiously, evidence of the ubiquitous gravitational fields consistent with a flat rotation curve also came 
from studies of elliptical galaxies, not only in the nearby universe (for NGC 4472, see lower left frame of Fig. 7 of Saglia et al. 1992; and Bertin 1993; for IC 2006, see Franx et al. 1994; for a more recent systematic study, see Gerhard et al. 2001), but also for ellipticals at cosmologically significant distances (e.g., see Barnabé et al. 2009, and references therein). The recent work by Gnedin et al. (2010) finds compatible evidence in the kinematics of the halo stars in the Milky Way out to $80 \mathrm{kpc}$.

Of course, deviations from the paradigmatic case of galaxies such as NGC 3198 are not uncommon (e.g., see Casertano \& van Gorkom 1991). But it has been pointed out that such deviations are generally associated with well-defined features in the distribution of visible matter or the noncircular motions (Sancisi 2004). On the other hand, pure disks characterized by the absence of specific photometric features are quite common (see also Kautsch 2009).

The literature on the general topic of the distribution of dark matter in spiral galaxies is enormous and deals with a variety of important aspects of the problem. In recent years these studies have generally focused on the requirements of current cosmological scenarios. In this Introduction it would be impossible to do justice to all the relevant studies in this general research area. However, it appears that one simple aspect of the problem has gone practically unnoticed (but see some discussion given by van Albada \& Sancisi 1986). This is the issue of the mutual interactions between halo and visible matter in determining the self-consistent equilibrium of the basic state. In their review, van Albada \& Sancisi (1986) addressed this point, with the help of $N$-body simulations, but did not carry out a systematic investigation.

Here we wish to reconsider this problem, starting from an extremely simple physical (and somewhat academic) description of the dark halo. We will thus address the problem of determining the nonspherical structure of a dark halo, assumed to be perfectly isothermal, in the presence of a given disk. We will show that even if very simple, and thus lacking some important features present in real objects (for example, the presence and role of gas are not considered explicitly in this paper, although they could be easily incorporated), this study leads to some interesting and unexpected results.

The search for equilibrium solutions of the Vlasov-Poisson equations, where the mean potential is generated by the distribution of stars populating the system, with the possible contribution of an "external" density distribution (e.g., associated with a co-existing dark matter halo), is a classical problem of stellar dynamics, with important analogues in the study of collisionless plasmas. In this paper we follow the approach based on the Jeans theorem to deal with the halo matter, while the disk of visible matter is taken to provide an external field. In this approach, the starting point is the identification of an appropriate form for the one-particle distribution function in phase space; in our case this is the isothermal assumption for the dark halo. The adopted distribution function then generates a $\Phi$-dependent density in physical space, thus leading, inserted as a source in the Poisson equation, to a nonlinear problem in $\Phi$. Here $\Phi$ represents the gravitational potential. In the past, this method has been used frequently and has led to important insights into the dynamics of galaxies and other stellar systems. Yet the study of self-consistent equilibria in the presence of external fields that break the natural symmetry associated with the one-component problem is only rarely considered (see Bertin \& Varri 2008). Therefore, besides the natural application to the discussion of the structure of dark halos around galaxies, this paper has some interest of its own, as a study in stellar dynamics.
The structure of the paper is the following. In Sect. 2 we comment on the so-called disk-halo conspiracy. Here we identify an empirical correlation which brings out a new and stronger conspiracy than the one associated with the flatness of rotation curves. In Sect. 3 we formulate the self-consistent problem of constructing an isothermal spheroid in the presence of a zero-thickness disk. The actual procedure to calculate the selfconsistent models is reported in Appendix A. The properties of self-consistent isothermal halos are then described in Sect. 4. Then in Sect. 5, by modeling first a fiducial rotation curve and later by modeling the specific case of NGC 3198, we show that self-consistency actually removes the degeneracy that characterizes standard parametric disk-halo decompositions (with additional comments given separately in Appendix B). Finally, Sect. 6 summarizes the main results of the paper. This article is all based on the mathematically simple case of zero-thickness disks. A follow-up paper will address the corresponding study of disks with finite thickness.

\section{A comment on the disk-halo "conspiracy"}

The rotation curve $V(R)$ of many spiral galaxies is featureless, regular, and smooth: after a central region of approximately linear growth, the rotation velocity reaches a constant value $V_{\infty}$ and remains flat out to large radii, often well beyond the bright optical disk. (But, of course, this is meant to be only a zero-th order description, because even flat rotaton curves are not entirely featureless, Sancisi \& van Albada 1987.) Since the underlying gravitational field results from the sum of the contributions of different mass components of the system (the stellar disk, the bulge, the gaseous disk, and the dark matter halo), which generally dominate in different regions, the smoothness and lack of features of the rotation curve is unexpected and poses a physical problem, which is commonly referred to as the disk-halo conspiracy.

When such conspiracy was noted (see van Albada \& Sancisi 1986) most of the emphasis was placed on the flatness of the rotation curves. In reality, it has long been noted that standard parametric disk-halo decompositions, based on the superposition of the fields produced by the visible matter and by a spherical halo, are subject to a high level of degeneracy. Equally viable fits to the rotation curves are obtained with very different models (i.e. very different values of the mass-to-light ratio of the stellar disk, assumed to be constant within the galaxy), ranging from some characterized by a very light disk and a dominant halo all the way in, to others, close to the so-called maximum disk solution, where the support to the rotation curve inside the optical disk is basically due to the visible matter alone, while the halo contribution becomes important and eventually dominates only outside. In a sense, the existence of such degeneracy actually undermines, or at least softens, the problem of conspiracy apparently raised by the flatness of rotation curves.

Of course, there are several constraints that can be used to reduce the level of degeneracy present. In addition to those posed by stellar population analyses, we may recall the constraints posed by dynamical stability in the context of spiral structure (Ostriker \& Peebles 1973; Bertin \& Lin 1996; see also Widrow et al. 2008, and references therein) or warp dynamics (e.g., see Bertin \& Mark 1980).

To give a concrete example of the procedure adopted in standard parametric disk-halo decompositions, we may recall the case of a purely exponential disk with the adoption of the model of a classical pseudo-isothermal sphere for the halo 
contribution. In this case the rotation curve is decomposed into $V_{\text {mod }}^{2}=V_{\mathrm{DM}}^{2}+V_{\mathrm{D}}^{2}$. Here

$V_{\mathrm{D}}^{2}(\xi)=V_{\infty}^{2} \frac{\beta}{8} \xi^{2}\left[I_{0}\left(\frac{\xi}{2}\right) K_{0}\left(\frac{\xi}{2}\right)-I_{1}\left(\frac{\xi}{2}\right) K_{1}\left(\frac{\xi}{2}\right)\right]$

where $\xi=R / h$ is the galactocentric cylindrical radius in units of the exponential scalelength and $\beta$ is a dimensionless parameter (see Eq. (16)) which measures the weight of the stellar disk. In addition, $I_{0}, K_{0}, I_{1}$, and $K_{1}$ denote the standard modified Bessel functions. The halo contribution is described by

$V_{\mathrm{DM}}^{2}(\xi)=V_{\infty}^{2}\left[1-\sqrt{\frac{2}{\alpha}} \frac{1}{\xi} \arctan \left(\sqrt{\frac{\alpha}{2}} \xi\right)\right]$,

where $\alpha$ is a dimensionless measure of the central dark matter density (see Eq. (19)). In this example, the degeneracy occurs in the $(\alpha, \beta)$ parameter space (see Sect. 5).

In the following subsection we will show the existence of a much stronger reason for invoking a problem of conspiracy, associated with the fine tuning between the length scale that characterizes the inner growth of the rotation curve and the natural scale length of the visible disk (in the absence of significant contributions from a bulge or from gas, this would be the exponential length). This tuning is a direct indication of a subtle interplay between the density distribution of dark and visible matter. If the two length scales were not sufficiently close to the observed correlation, the degeneracy of the standard parametric disk-halo decompositions would not take place.

\subsection{An important empirical correlation}

This subsection will provide a useful empirical background to the physically simple dynamical models presented in this paper. Here we start by identifying one important aspect of the problem of the uniformity of the observed characteristics of rotation curves.

For simplicity, we refer to a sample of galaxies for which the contribution of the bulge should be negligible. We first consider disk galaxies belonging to the Ursa Major cluster, for which good-quality photometric profiles (Tully et al. 1996) and rotation curves (Verheijen \& Sancisi 2001) are available. Without actually aiming at producing a complete sample in any statistically significant sense, which would go well beyond the goals of this paper, we then add a small number of other galaxies, with data taken from Kent (1987), de Blok \& McGaugh (1997) (with photometries from de Blok et al. 1995), and Swaters et al. (2000; with photometries from de Blok et al. 1995; and from McGaugh \& Bothun 1994), so as to increase the size of the sample. The only criterion used to select the galaxies from the source papers is the shape of their photometric profiles. Galaxies with clear deviations from an exponential profile in the central parts, often related to the presence of a bulge, are discarded. Therefore, for these approximately exponential stellar disks, any difference in the shape of the rotation curves should be interpreted only in terms of the different relative weights of the disk and of the dark component (under the simplifying assumption that the mass-to-light ratio is constant within each galaxy disk). The final sample (see Table 1) thus consists of thirty-five objects, almost equally divided between High Surface Brightness and Low Surface Brightness galaxies.

For each galaxy we consider the exponential length $h$ of the stellar disk and the radius $R_{\Omega}$ that we define as the radius
Table 1. The final sample.

\begin{tabular}{|c|c|c|c|c|}
\hline Name & Type & $h / \mathrm{kpc}$ & $R_{\Omega} / \mathrm{kpc}$ & Refs. \\
\hline NGC 247 & HSB & 2.7 & $3.5 \pm 0.6$ & [4] \\
\hline NGC 300 & HSB & 1.8 & $1.9 \pm 0.3$ & [4] \\
\hline NGC 2403 & HSB & 2.1 & $1.8 \pm 0.3$ & [4] \\
\hline NGC 3109 & HSB & 1.35 & $1.6 \pm 0.3$ & [4] \\
\hline NGC 3769 & HSB & 1.5 & $2.2 \pm 0.8$ & {$[1],[2]$} \\
\hline NGC 3877 & HSB & 2.4 & $2.6 \pm 0.4$ & [1], [2] \\
\hline NGC 3893 & HSB & 2.0 & $1.7 \pm 0.4$ & [1], [2] \\
\hline NGC 3972 & HSB & 1.6 & $2.5 \pm 0.5$ & {$[1],[2]$} \\
\hline NGC 3917 & LSB & 2.6 & $2.6 \pm 0.2$ & [1], [2] \\
\hline NGC 4010 & LSB & 2.9 & $3.8 \pm 0.8$ & [1], [2] \\
\hline NGC 4085 & HSB & 1.3 & $1.9 \pm 0.3$ & [1], [2] \\
\hline NGC 4157 & HSB & 2.2 & $2.4 \pm 0.5$ & [1], [2] \\
\hline NGC 4183 & LSB & 2.65 & $2.9 \pm 0.8$ & [1], [2] \\
\hline NGC 4217 & HSB & 2.4 & $2.4 \pm 0.5$ & [1], [2] \\
\hline NGC 4236 & HSB & 2.7 & $3 \pm 0.4$ & [4] \\
\hline NGC 4389 & HSB & 1.2 & $1.9 \pm 0.9$ & [1], [2] \\
\hline UGC 128 & LSB & 6.8 & $7 \pm 1.5$ & [3], [5] \\
\hline UGC 1230 & LSB & 4.5 & $6 \pm 1.2$ & {$[3],[5]$} \\
\hline UGC 5005 & LSB & 4.4 & $4.5 \pm 0.6$ & [3], [5] \\
\hline UGC 5750 & LSB & 5.6 & $5.8 \pm 1$ & [3], [5] \\
\hline UGC 5999 & LSB & 4.3 & $5 \pm 1$ & [3], [5] \\
\hline UGC 6399 & LSB & 2.0 & $2.1 \pm 0.4$ & [1], [2] \\
\hline UGC 6446 & LSB & 1.5 & $1.6 \pm 0.6$ & [1], [2] \\
\hline UGC 6667 & LSB & 2.45 & $2.3 \pm 0.2$ & [1], [2] \\
\hline UGC 6917 & LSB & 2.4 & $2.3 \pm 0.2$ & {$[1],[2]$} \\
\hline UGC 6923 & LSB & 1.1 & $1.8 \pm 0.2$ & [1], [2] \\
\hline UGC 6930 & LSB & 2.0 & $2.4 \pm 0.3$ & [1], [2] \\
\hline UGC 6962 & HSB & 1.4 & $1.6 \pm 0.5$ & [1], [2] \\
\hline UGC 6983 & LSB & 2.2 & $1.9 \pm 0.5$ & [1], [2] \\
\hline F 561-1 & LSB & 2.8 & $2.8 \pm 0.5$ & [3], [5] \\
\hline F 563-V2 & LSB & 2.1 & $1.8 \pm 0.3$ & [7], [6] \\
\hline F 565-V2 & LSB & 2.7 & $3.5 \pm 0.5$ & [3], [5] \\
\hline F 568-3 & LSB & 4.0 & $3.1 \pm 0.5$ & [3], [6] \\
\hline F 568-V1 & LSB & 3.2 & $2.7 \pm 0.4$ & [3], [6] \\
\hline F 571-V1 & LSB & 3.2 & $4 \pm 1$ & {$[3],[5]$} \\
\hline
\end{tabular}

References. The references are coded as: [1] Tully et al. (1996), [2] Verheijen \& Sancisi (2001), [3] de Blok et al. (1995), [4] Kent (1987), [5] de Blok \& McGaugh (1997), [6] Swaters et al. (2000), [7] McGaugh \& Bothun (1994).

at which the rotation curve reaches two thirds of its estimated asymptotic flat value $\hat{V}_{\infty}$ :

$V\left(R_{\Omega}\right)=\frac{2}{3} \hat{V}_{\infty}$

The velocity $\hat{V}_{\infty}$ is the estimate of the asymptotic velocity $V_{\infty}$ obtained by averaging the rotation velocity in the outer parts of the rotation curve (see also Sect. 4.2). The radius $R_{\Omega}$ thus measures the slope of the inner approximately linear growth of the rotation curve.

When available (for example in the whole sample of galaxies belonging to the Ursa Major cluster), the reported exponential length is relative to the $K$-band photometry of the stellar disk, which is known to be the best representation of the actual stellar density distribution. In all the other cases we use the data as reported in the original source papers. We do not consider explicit error estimates for the exponential lengths $h$, since a uniform estimate would not be available for all the galaxies in the sample. The estimate for the radius $R_{\Omega}$ is calculated directly, following its definition Eq. (3), from the observed rotation curve profile. The relevant error is estimated here by considering the errors in the velocity datapoints and the process of interpolating the rotation curve between its measured points. 


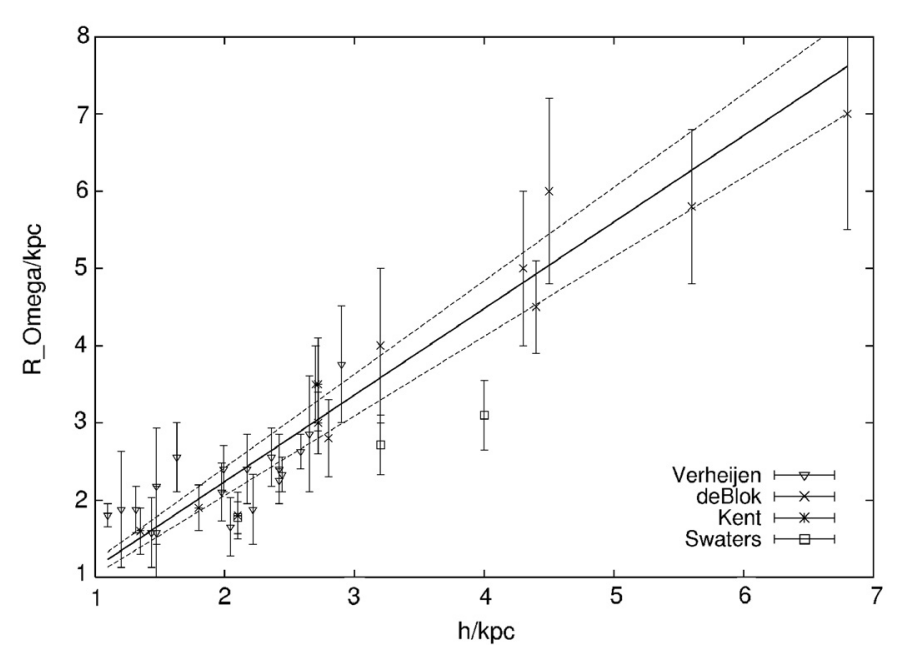

Fig. 1. Correlation between the characteristic lengths $h$ and $R_{\Omega}$ (see text for a description) for the sample of disk galaxies of Table 1 . The dashed lines represent $3 \sigma$ error on the best-fit value of the angular coefficient.

Figure 1 demonstrates that there is indeed a strong, tight correlation between the two length scales considered. The correlation found is

$R_{\Omega} / h=1.07 \pm 0.03$.

The error on this measure is the standard statistical error, calculated by taking into account the uncertainties assigned to the values of $R_{\Omega}$.

Given the success of this test, it would be interesting to carry out a similar investigation on a better-defined, "cleaner" sample of galaxies, so as to test how much of the scatter around the correlation is intrinsic or rather due to the quality of the available data. However, a discussion of this point would bring us away from the main objective of this paper.

The correlation coefficient for the entire sample is $r_{h-R_{\Omega}}=$ 0.96. It is thus interesting to note that the same correlation applies to both HSB and LSB galaxies. The correlation provides additional support to a conspiracy argument, because it shows that even in the inner region of approximately linear growth of the rotation curve the dark matter distribution must be in tune with the distribution of the visible mass. Note that this is not necessarily in favor of the maximum-disk hypothesis, but rather it suggests that the relative distribution of visible and dark matter in spiral galaxies is homologous among different objects. Such homology could also be at the basis of observed correlations such as the Tully-Fisher (1977) relation (van Albada \& Sancisi 1986).

In addition, this result will allow us to make a significant (although preliminary) test of the models developed in this paper on a simple idealized prototypical case that incorporates the observed correlation, without the need for separate tests on several observed objects.

\section{An isothermal halo embedding a zero-thickness disk}

We refer to standard cylindrical coordinates $(R, z, \theta)$, so that $r=\sqrt{R^{2}+z^{2}}$ represents the spherical radius. We assume that the rotation curve $V(R)$ of the disk galaxy, for which we want to construct a mass model, is known (i.e., it has been "observed"); as a mathematical boundary condition, we assume that the rotation curve remains flat at $V_{\infty}$ out to infinite radii.
In order to make a fit to the observed rotation curve, we wish to construct axisymmetric equilibrium models made of two mass components: the visible disk and the dark halo. For simplicity, we imagine the disk to be made only of stars, with known surface brightness profile $\mu(R)$, and ignore the contribution of other visible components. It will soon be clear that the contributions from a gaseous disk and from a spheroidal bulge with given structure could be easily incorporated, if desired. The visible disk is described as a zero-thickness disk; in a follow-up paper, we will address the study of a self-consistent finite-thickness disk as a generalization of the present model.

The dark halo is taken to be characterized by a Maxwellian, isothermal distribution function. The choice of the dark matter distribution function is justified by an argument of physical simplicity. With the minimum number of free parameters, it guarantees the flatness of the outer parts of the rotation curve. The procedure that we are going to describe can be easily adapted to study dark halos characterized by other physically justified distribution functions, if so desired (e.g., see Bertin et al. 1992; and Bertin 2000). In conclusion, for the dark matter halo we take the isotropic distribution function:

$f_{\mathrm{DM}}(E)=\frac{\rho_{\mathrm{DM}}^{0}}{\left(2 \pi \sigma^{2}\right)^{3 / 2}} \exp \left(-E / \sigma^{2}\right)$,

where $E=v^{2} / 2+\Phi_{\mathrm{T}}$ is the specific energy of dark matter particles and $\Phi_{\mathrm{T}}$ is the total gravitational potential. In our model $\Phi_{\mathrm{T}}$ is determined by the dark matter halo and by the disk:

$\Phi_{\mathrm{T}}=\Phi_{\mathrm{DM}}+\Phi_{\mathrm{D}}$

The density distribution associated with the distribution function (5) is

$\rho_{\mathrm{DM}}(R, z)=\int \mathrm{d}^{3} \boldsymbol{v} f_{\mathrm{DM}}=\rho_{\mathrm{DM}}^{0} \exp \left[-\Phi_{\mathrm{T}}(R, z) / \sigma^{2}\right]$,

where we have made it explicit that, in general, it is not spherically symmetric, because of the presence of the disk. At large radii, the gravitational contribution of the disk becomes vanishingly small, so that the total gravitational potential eventually approaches the natural spherical symmetry of the self-consistent isolated isothermal sphere.

The dimensional free scales of the distribution function in Eq. (5) are the central density $\rho_{\mathrm{DM}}^{0}$ and the (constant) velocity dispersion $\sigma$. We identify the quantity $\rho_{\mathrm{DM}}^{0}$ with the central dark matter density, because we impose the condition

$\Phi_{\mathrm{T}}(0,0)=0$.

In other words, we will only consider models that are regular at the center.

The velocity dispersion $\sigma$ is uniquely fixed by the value $V_{\infty}$ of the rotation curve at large radii, so that the central dark matter density $\rho_{\mathrm{DM}}^{0}$ is the only remaining free dimensional scale for the adopted dark matter density distribution. In fact, at large distances from the center, where the system is almost spherically symmetric, matching with the observed rotation curve requires

$\Phi_{\mathrm{T}}(R, z) \sim \Phi_{\mathrm{T}}(r) \sim V_{\infty}^{2} \ln \left(\frac{r}{r_{0}}\right)$,

which, inserted in the Poisson equation, gives

$$
\left\{\begin{array}{l}
V_{\infty}^{2}=2 \sigma^{2} \\
V_{\infty}^{2}=4 \pi G \rho_{\mathrm{DM}}^{0} r_{0}^{2} .
\end{array}\right.
$$


We thus see that the scale length $r_{0}$ of the dark matter density distribution appearing in Eq. (9) is not a free length, because for assigned value of $V_{\infty}$ (i.e., of $\sigma$ ) it is determined by the value of the central dark matter density, $\rho_{\mathrm{DM}}^{0}$.

For our zero-thickness disk, we take the surface density to be proportional to the observed surface brightness $\mu(R)$, i.e.:

$\rho_{\mathrm{D}}(R, z)=\left(\frac{M}{L}\right) \mu(R) \delta(z)$;

here the function $\delta$ is the Dirac delta-function. The ratio $M / L$ is the mass-to-light ratio of the stellar disk, which we take to be constant; for assigned photometric profile $\mu(R)$, it represents the only free dimensional scale of the stellar density distribution.

The model is constructed by solving the complete Poisson equation for the system, in which the matter densities are defined by the distributions (7) and (11):

$\nabla^{2} \Phi_{\mathrm{T}}(R, z)=4 \pi G\left[\rho_{\mathrm{D}}(R, z)+\rho_{\mathrm{DM}}(R, z)\right]$.

This is a nonlinear differential equation for the total gravitational potential $\Phi_{\mathrm{T}}$, which must be solved with the appropriate boundary conditions: Eq. (8), as the condition at the center, and Eq. (9), for the asymptotic behavior at large radii. The solution of the Poisson Eq. (12), which depends on the two free scales $\rho_{\mathrm{DM}}^{0}$ and $M / L$, will allow us to calculate the rotation curve of the model $V_{\text {mod }}(R)=\sqrt{\left.R\left(\partial \Phi_{\mathrm{T}} / \partial R\right)\right|_{z=0}}$, to be compared with the observed $V(R)$.

Note that this method is intrinsically different from the standard parametric decompositions of the mass models of spiral galaxies. In standard parametric analyses, the contribution of the dark matter halo to the total gravitational potential has a fixed shape (usually, spherically symmetric), which is independent of the presence and gravitational importance of the stellar disk and which can be changed only through a set of parameters $a_{i}$, that is $\Phi_{\mathrm{DM}}=\Phi_{\mathrm{DM}}\left(R, z ; a_{i}\right)$. The present self-consistent models $d o$ not have a fixed parametric profile for the shape of the gravitational potential of the dark matter component. Through Eq. (12), the dark matter gravitational potential, and thus its contribution to the total rotation curve, depends implicitly also on the properties of the stellar disk (its surface brightness $\mu(R)$ and its mass to light ratio), that is $\Phi_{\mathrm{DM}}=\Phi_{\mathrm{DM}}\left(R, z ; \rho_{\mathrm{DM}}^{0} ; \mu(R), M / L\right)$.

In conclusion, for a given rotation curve $V(R)$ and surface brightness of the stellar disk $\mu(R)$, the best-fit self-consistent disk-halo decomposition will correspond to the self-consistent model defined by the pair $\left(\rho_{\mathrm{DM}}^{0}, M / L\right)$, together with the asymptotic velocity $V_{\infty}$, that gives the best fit to the observed rotation curve.

\subsection{The mathematical problem in dimensionless form}

As units of length and of specific energy we will consider the scale length of the stellar disk $h$ and the square of the velocity dispersion $\sigma^{2}=V_{\infty}^{2} / 2$, respectively, so that we introduce the dimensionless coordinates

$\xi \equiv R / h, \zeta \equiv z / h, \eta \equiv r / h$

and the dimensionless gravitational potential

$\psi_{\mathrm{T}}=\psi_{\mathrm{DM}}+\psi_{\mathrm{D}} \equiv-\Phi_{\mathrm{T}} /\left(V_{\infty}^{2} / 2\right)$.

We separate the Poisson Eq. (12) in the two Poisson equations for the two different mass components of the system. The dimensionless equation for the stellar disk reads

$\left(\frac{1}{\xi} \frac{\partial}{\partial \xi} \xi \frac{\partial}{\partial \xi}+\frac{\partial^{2}}{\partial \zeta^{2}}\right) \psi_{\mathrm{D}}=-\frac{\beta}{2} \hat{\Sigma}(\xi) \delta(\zeta)$.
Here we have introduced the normalized surface density, defined by $\hat{\Sigma}(R) \equiv \Sigma(R) / \Sigma(0)$, and the parameter $\beta$

$\beta \equiv \frac{8 \pi G \Sigma(0) h}{V_{\infty}^{2}}=\frac{M}{L} \frac{8 \pi G \mu(0) h}{V_{\infty}^{2}}$,

which is the dimensionless version of the weight of the stellar disk as measured by the mass-to-light ratio $M / L$.

To solve Eq. (15), we may use the standard technique of Hankel transforms (see Toomre 1963). In this way the potential associated with any surface density profile $(M / L) \mu(R)$ can be calculated exactly. Consistent with the condition (8), we also impose that $\psi_{\mathrm{D}}(0,0)=0$. In particular, for purely exponential disks, $\hat{\Sigma}=\exp (-\xi)$, the associated gravitational potential is given by

$\psi_{\mathrm{D}}(\xi, \zeta)=-\frac{\beta}{2}\left\{\int_{0}^{\infty} \mathrm{d}(h k) \frac{\exp (-h k|\zeta|) J_{0}(h k \xi)}{\left[1+(h k \xi)^{2}\right]^{3 / 2}}-1\right\}$,

where $J_{0}$ follows the standard notation of Bessel functions.

In turn, the Poisson equation for the dark matter halo can be written as

$\left(\frac{1}{\xi} \frac{\partial}{\partial \xi} \xi \frac{\partial}{\partial \xi}+\frac{\partial^{2}}{\partial \zeta^{2}}\right) \psi_{\mathrm{DM}}=-\alpha \exp \left[\psi_{\mathrm{DM}}(\xi, \zeta)+\psi_{\mathrm{D}}(\xi, \zeta)\right]$

where the parameter $\alpha$ can be seen as the dimensionless expression of the central dark matter density scale:

$\alpha \equiv \frac{8 \pi G h^{2} \rho_{\mathrm{DM}}^{0}}{V_{\infty}^{2}}=2 \frac{h^{2}}{r_{0}^{2}}$

The parameter $\alpha$ measures the halo concentration. With an eye to the central region of the system, a measure of the halo mass relative to the the mass of the disk is given by the parameter $\alpha / \beta=\rho_{\mathrm{DM}}^{0} h / \Sigma_{0}$ (see also Eq. (12)). Thus the dimensional pair $\left(\rho_{\mathrm{DM}}^{0}, M / L\right)$ has its dimensionless counterpart in $(\alpha, \beta)$. In the $(\alpha, \beta)$ plane, the straight lines defined by $\beta \propto \alpha$ can be interpreted as lines of approximately given central density of the halo with respect to the mass of the disk; steep lines are those characterizing relatively light halos. A better defined measure of the weight of the halo will be given in the next section.

The Poisson Eq. (18) for the dark matter halo has to be solved numerically (see Appendix A). Here the gravitational potential of the disk, calculated separately as described above, acts as an external potential that breaks the spherical symmetry and shapes the dark matter density distribution. The relevant boundary conditions can be obtained from the boundary conditions imposed on the total gravitational potential:

$$
\left\{\begin{array}{l}
\psi_{\mathrm{DM}}(0,0)=0 \\
\psi_{\mathrm{DM}}(\eta) \sim-2 \ln \left(\eta \sqrt{\frac{\alpha}{2}}\right)-\psi_{\mathrm{D}}^{\infty}, \eta \gg 1,
\end{array}\right.
$$

where the symbol $\psi_{\mathrm{D}}^{\infty}$ indicates the constant value approached (with spherical symmetry) by the dimensionless gravitational potential of the disk at large distances from the center of the system.

\section{Properties of self-consistent spheroidal isothermal halos}

The structure of the dark matter halo reflects the structure of the total gravitational potential, as prescribed by Eq. (7). 


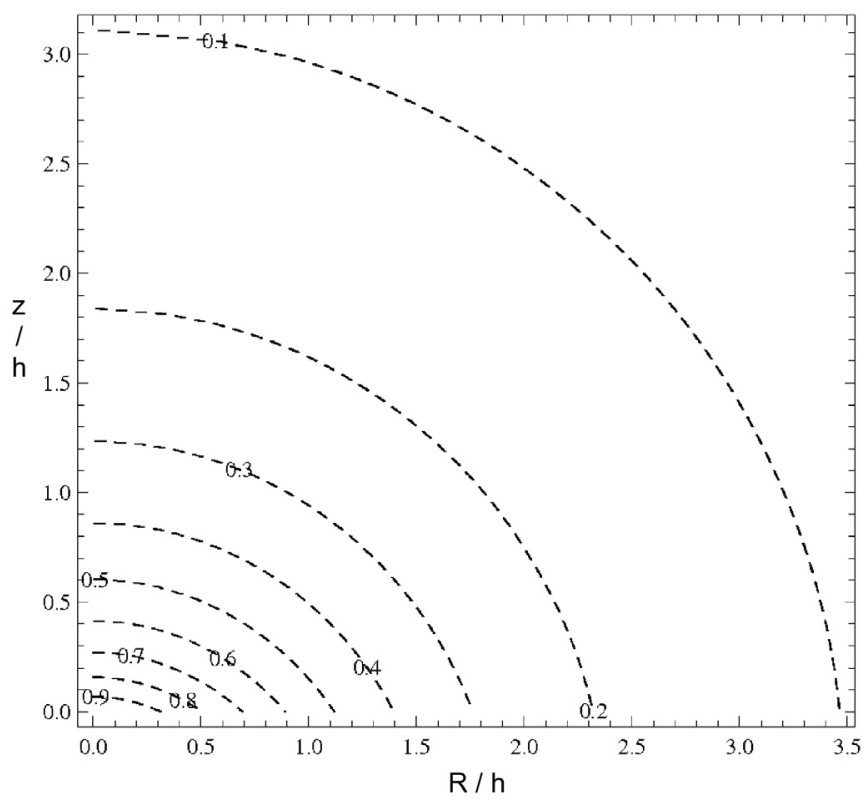

Fig. 2. Contours of the dark halo density distribution (normalized to its central value) for the model defined by Eq. (30). Note that these contours coincide with those of the total gravitational potential.

On the one hand, there is an artificial cusp in the vertical dark matter density distribution on the $z=0$ plane, which results from the singular (zero-thickness) density distribution assumed for the stellar disk. The detailed structure of the cusp can be obtained from the Jeans equation:

$$
\frac{\sigma^{2}}{\rho_{\mathrm{DM}}(R, 0)}\left[\frac{\partial}{\partial z} \rho_{\mathrm{DM}}\left(R, 0^{+}\right)-\frac{\partial}{\partial z} \rho_{\mathrm{DM}}\left(R, 0^{-}\right)\right]=\left.\frac{\partial \Phi_{\mathrm{D}}}{\partial z}\right|_{\left(R, 0^{-}\right)} ^{\left(R, 0^{+}\right)} .
$$

This feature has no physical relevance and will be naturally eliminated in the finite-thickness disk models that we plan to present in a separate paper.

On the other hand, the halo is characterized by significant flattening. This latter feature is genuine and will not disappear in the regular finite-thickness case. Figure 2 shows the contours of the total gravitational potential, which coincide with those of the dark matter density, for the model defined by $(\alpha, \beta)=(1.9,3.4)$, which, as we will see in the following, is the self-consistent model representative of the correlation in Eq. (4).

\subsection{The shape of isothermal halos}

As naturally expected, the system tends to be spherically symmetric in the outer parts (where the gravitational importance of the disk rapidly vanishes), but is highly oblate in the central regions. The flattening of the dark halo can be quantified in terms of the ellipticity $\varepsilon$, defined as

$\varepsilon(\xi)=1-\frac{\nu(\xi)}{\xi}$,

where $\xi$ and $v(\xi)$ represent respectively the intersections with the $\zeta=0$ and $\xi=0$ planes of a given contour of the total gravitational potential. The profile of the ellipticity $\varepsilon$ as a function of radius is illustrated in Fig. 3. The high flattening inside one exponential length may be exaggerated because of the zero-thickness of the disk. Figure 4 shows the ellipticity reached by the selfconsistent models at 2.2 exponential lengths in the $(\alpha, \beta)$ plane.

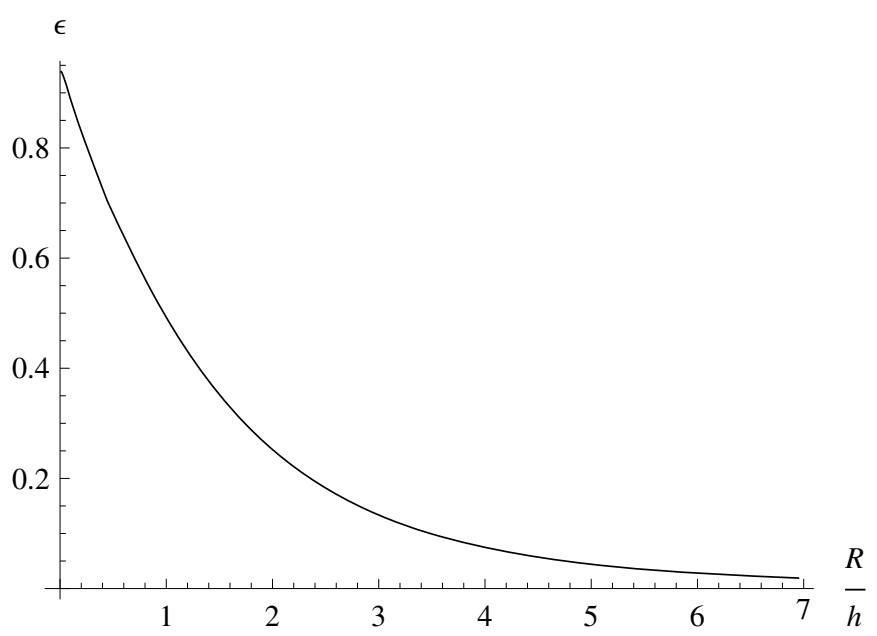

Fig. 3. Ellipticity profile of the dark matter halo illustrated in Fig. 2, as defined by Eq. (22).

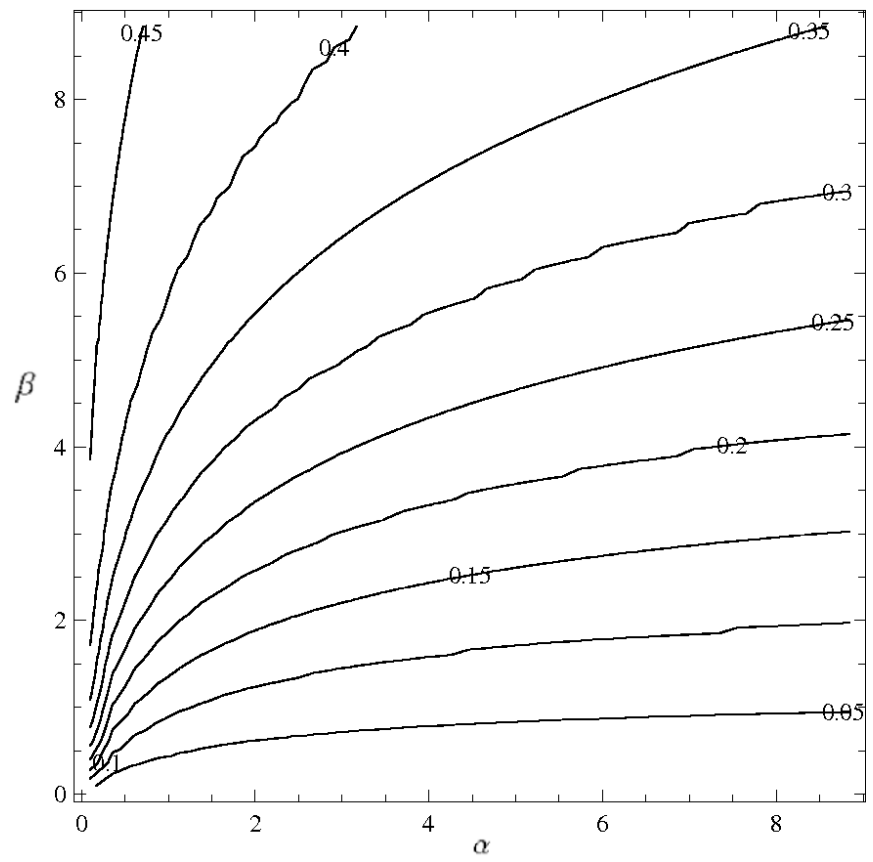

Fig. 4. The ellipticity at 2.2 exponential lengths $\varepsilon(2.2)$ in the $(\alpha, \beta)$ plane.

It would be interesting to compare the flattening obtained from our self-consistent models with the empirical determinations based on a variety of dynamical arguments, ranging from the dynamics of warped disks and polar rings to the flaring of gas layers (see the discussion in the paper by Olling \& Merrifield 2000; see also the series of papers started by O'Brien et al. 2010). It would also be interesting to compare the flattening of our self-consistent models with the indications that are expected to be gathered in the near future from weak and strong gravitational lenses (for example, see Trott et al. 2010); here, the natural comparison to be made is in terms of the relevant flattening profile of the projected density distribution.

\subsection{The flatness of self-consistent rotation curves}

A significant feature of the self-consistent models presented in this paper is the unexpected degree of flatness that characterizes 
their rotation curves. In principle, self-consistent rotation curves are designed to be flat only as an asymptotic condition for $R \gg h$, that is where the gravitational influence of the stellar disk becomes negligible. In the intermediate radial range the shape of the rotation curve is instead determined by the mutual interactions between halo and visible disk and thus cannot be ascribed naively to the Maxwellian form of the halo distribution function (especially because in the intermediate radial range an isothermal sphere is known to be associated with a rotation curve exhibiting a characteristic oscillation pattern).

To better demonstrate this point and to clarify the origin of the observed flatness, the self-consistent rotation curves will be compared with those of two different non-self-consistent models: (i) the parametric models, defined by Eqs. (1), (2); (ii) the models, called for short semi-parametric, for which the field of the disk, with associated rotation curve defined by Eq. (1), is superposed (non-self-consistently) to the field of a spherical isothermal halo, i.e. a halo described by the density distribution in Eq. (7), in which the total potential $\Phi_{\mathrm{T}}(R, z)$ is replaced by the dark matter potential $\Phi_{\mathrm{DM}}(r)$. Therefore, any difference in the rotation curves between semi-parametric models and self-consistent models must be ascribed to the effects of selfconsistency alone.

With the aim of providing a systematic and quantitative analysis, we start by defining as intermediate radial range the range $2.2 h<R<7 h$, i.e. $2.2<\xi<7$. Here the lower limit corresponds to the location at which the rotation curve of an isolated exponential disk reaches its maximum, while the upper limit represents a reasonable choice of a location outside the bright optical disk. The quantity $\hat{V}_{\infty}$ will denote the average value of the rotation curve $V_{\text {mod }}$ in the outer parts, $4<\xi<7$. We then introduce the function

$\Theta(\alpha, \beta)=\int_{2.2}^{7}\left|\frac{1}{\hat{V}_{\infty}} \frac{\partial V_{\text {mod }}}{\partial \xi}\right| \mathrm{d} \xi$.

The function $\Theta$ is constructed so as to measure the flatness of the rotation curve $V_{\text {mod }}$ in the intermediate radial range. The $\Theta$-contours calculated from the self-consistent models are displayed in Fig. 5. In Fig. 6 the two left panels show the contours of the function $\Theta$ obtained from the parametric models (upper frame) and the semi-parametric models (lower frame), for the same region of parameter space. A set of representative contours has been chosen arbitrarily, with the aim of describing the properties of the three different cases.

With respect to the range of values of the function $\Theta$, the parametric models and the semi-parametric models differ significantly from each other, because the semi-parametric models exhibit rotation curves that are less flat over the entire region of parameter space. This behavior is due to the fact that regular isothermal spheres are characterized by a well-known oscillation pattern in their density distribution and in the associated rotation curve, so that in the inner region the rotation curve overshoots its asymptotic value (see also Fig. 9). This property is removed a priori in the parametric profile of Eq. (2), often adopted in this context. In contrast, self-consistent models show a degree of flatness comparable to that of parametric models. The right panels of Fig. 6 illustrate (upper frame) the contours of the ratio between the function $\Theta$ for parametric models and the same function for self-consistent models and (lower frame) the contours of the ratio between the function $\Theta$ for semi-parametric models and the same function for self-consistent models. It is then clear that, in a relatively wide region of parameter space, where the contour levels exceed unity in the lower right frame of Fig. 6,

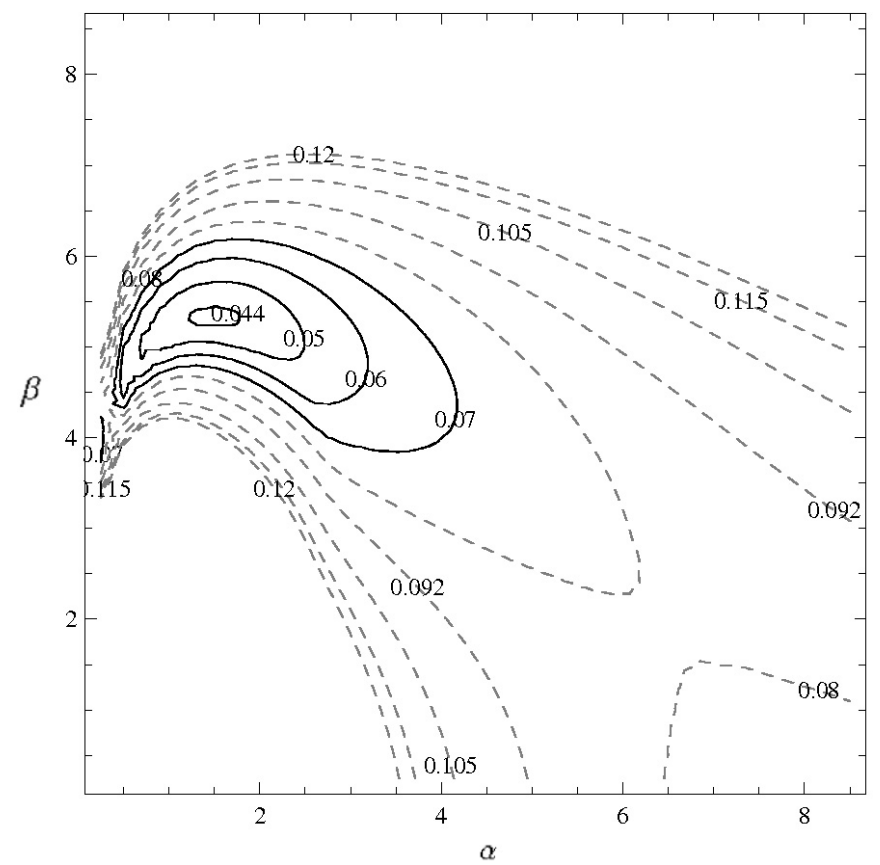

Fig. 5. Contours of the function $\Theta$ (Eq. (23)) for self-consistent models.

self-consistent rotation curves are more flat than the corresponding semi-parametric rotation curves; as stated previously, this flattening effect can only be due to self-consistency (this point will be addressed further in Sect. 4.4). In addition, there is a region of parameter space in which self-consistent rotation curves are more flat than parametric rotation curves (where the contour levels exceed unity in the upper right frame of Fig. 6). This latter comparison has no special physical meaning, but it confirms the high degree of flatness of self-consistent rotation curves (which is also evident from Fig. 8).

Another important point of comparison is the shape of the contours of the function $\Theta$ for the different cases. While the selfconsistent models are flat in a well-defined region of parameter space (e.g., see the distinction between solid and dashed contours in Fig. 5), the parametric models are uniformly flat over an entire diagonal strip of parameter space (upper left frame of Fig. 6). In Sect. 5 we will see that this strip is remarkably similar to the disk-halo degeneracy strip.

\subsection{The inner gradient of the self-consistent rotation curves}

After characterizing the properties of the self-consistent models at intermediate radii, in this subsection we focus on the properties of the inner regions. We start by defining the radius $R_{\Omega}$ from the relation

$V\left(R_{\Omega}\right)=\frac{2}{3} \hat{V}_{\infty}$

with $\hat{V}_{\infty}$ introduced in Sect. 4.2, Fig. 7 shows the contours of the quantity $R_{\Omega} / h$ for self-consistent models (black contours) and parametric models (gray contours). The thick contours in Fig. 7 represent the contours corresponding to the empirical correlation found in Eq. (4).

The general shape of the contours is significantly different for the two models. While self-consistent models show roundish contours, parametric models exhibit a clear diagonal trend, which reinforces the expectation that a degeneracy problem will 

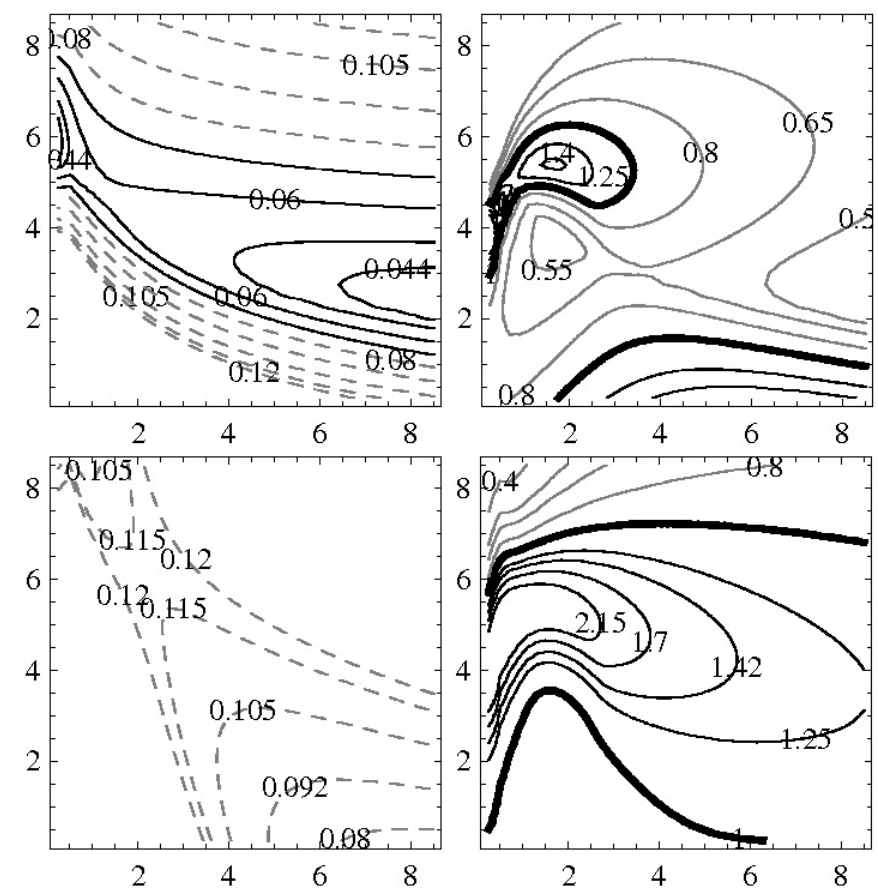

Fig. 6. Top-left: contours of the function $\Theta$ for parametric mass models. Top-right: contours of the ratio between the function $\Theta$ for parametric models and the same function for self-consistent models. Bottom-left: contours of the function $\Theta$ for semi-parametric models, with isothermal spherical halo. Bottom-right: contours of the ratio between the function $\Theta$ for semi-parametric models and the same function for self-consistent models. In the right frames, the thick contour represents unity. The axes in the four panels are $(\alpha, \beta)$, as in Fig. 5.

arise in the corresponding disk-halo decompositions, as noted in the previous subsection.

Finally, in the case of self-consistent models, the general shape of the $R_{\Omega} / h$ contours is similar to that of the contours of the function $\Theta$. The $R_{\Omega} / h$ value corresponding to maximumflatness is slightly below the one suggested empirically by the analysis of Sect. 2.1, that is it corresponds to a slightly faster growing rotation curve (but see also the case of NGC 3198, described in Sect. 5.2).

\subsection{Systematic properties of the self-consistent solutions in the available parameter space}

The aim of this subsection is to provide a description of the properties of self-consistent rotation curves in the relevant parameter space.

Figure 8 shows the rotation curves and the related diskhalo decompositions for an atlas of self-consistent models, in the region of parameter space of astrophysical interest (see also Sect. 5). In each frame the disk contribution to the rotation curve is dash-dotted, the contribution of the self-consistent halo is the solid black curve and the resulting rotation curve is indicated by a thick curve. Each frame records the pair $\left(M_{3.5}, M_{7.0}\right)$. The quantities $M_{3.5}$ and $M_{7.0}$ indicate the ratio of the halo mass to the disk mass inside the spheres with radii of 3.5 and 7 exponential lengths, respectively.

As already noted in Sect. 4.2, in general the rotation curves are significantly flat and lack specific features. The flattening effect described in Sect. 4.2 shows up clearly in Fig. 8, in a sort

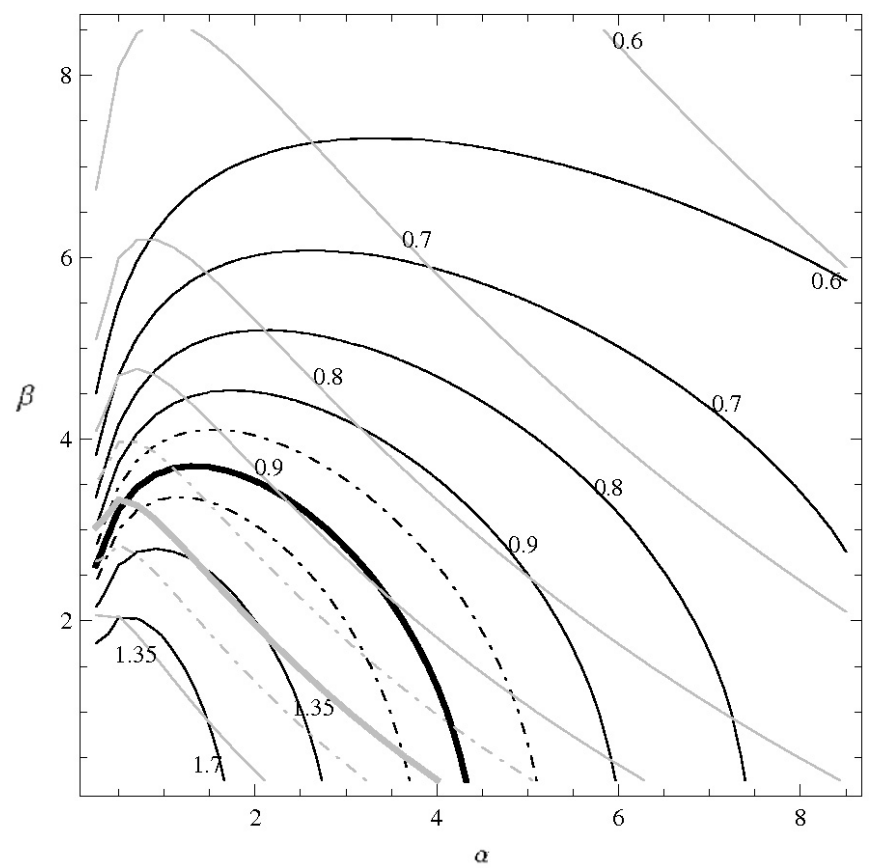

Fig. 7. Contours of the quantity $R_{\Omega} / h$, defined in Eq. (24). The gray contours, exhibiting a diagonal trend, are associated with the parametric models defined by Eqs. (1) and (2), while the black contours are associated with the self-consistent models. In both cases the thick line represents the empirical value found in Eq. (4), and the dashed lines represent the edges of the interval defined by a $3 \sigma$ deviation.

of suppression effect. Here the effects on the self-consistent halo caused by the presence of the stellar disk can be followed by looking at each individual column, that is by fixing the value of the dimensionless central density of the halo $\alpha$ and by letting the weight of the disk $\beta$ increase. The parameter $\alpha$ fixes the innermost gradient of the dark halo contribution to the rotation curve (which is proportional to $\sqrt{G \rho_{\mathrm{DM}}^{0}}$ ). However, in general selfconsistency cannot support a fast growing contribution from the dark halo in the presence of an important stellar disk, and then it "softens" it near $1 \div 2$ exponential lengths. At low values of $\beta$ self-consistency suppresses the bump in the halo contribution to the rotation curve characteristic of the isothermal sphere (e.g., compare the $(\alpha=9, \beta=0.5)$ frame to the $(\alpha=9, \beta=2)$ frame). For heavier disks (high values of $\beta$ ), self-consistency suppresses the halo contribution at intermediate radii (e.g., compare the ( $\alpha=0.5, \beta=3.5$ ) frame to frames corresponding to higher values of $\beta$ ). For high values of both $\alpha$ and $\beta$ (see for example $(\alpha=9, \beta=8)$ ) self-consistency makes the halo contribution fairly constant, but with a value which is significantly below $100 \%$, which will be reached eventually, at much larger radii.

This suppression effect has three main consequences on the general shape of self-consistent rotation curves: it flattens them globally (with respect to the rotation curves of the semiparametric models; see Sect. 4.2); it removes the oscillations characteristic of the isolated isothermal sphere (provided the stellar disk is not too light); it makes the signature of an important luminous disk more evident, by lowering the halo contribution.

Similar general conclusions can be made by inspection of Fig. 9. Total rotation curves of self-consistent models (thick gray lines), parametric models (black solid lines), and 


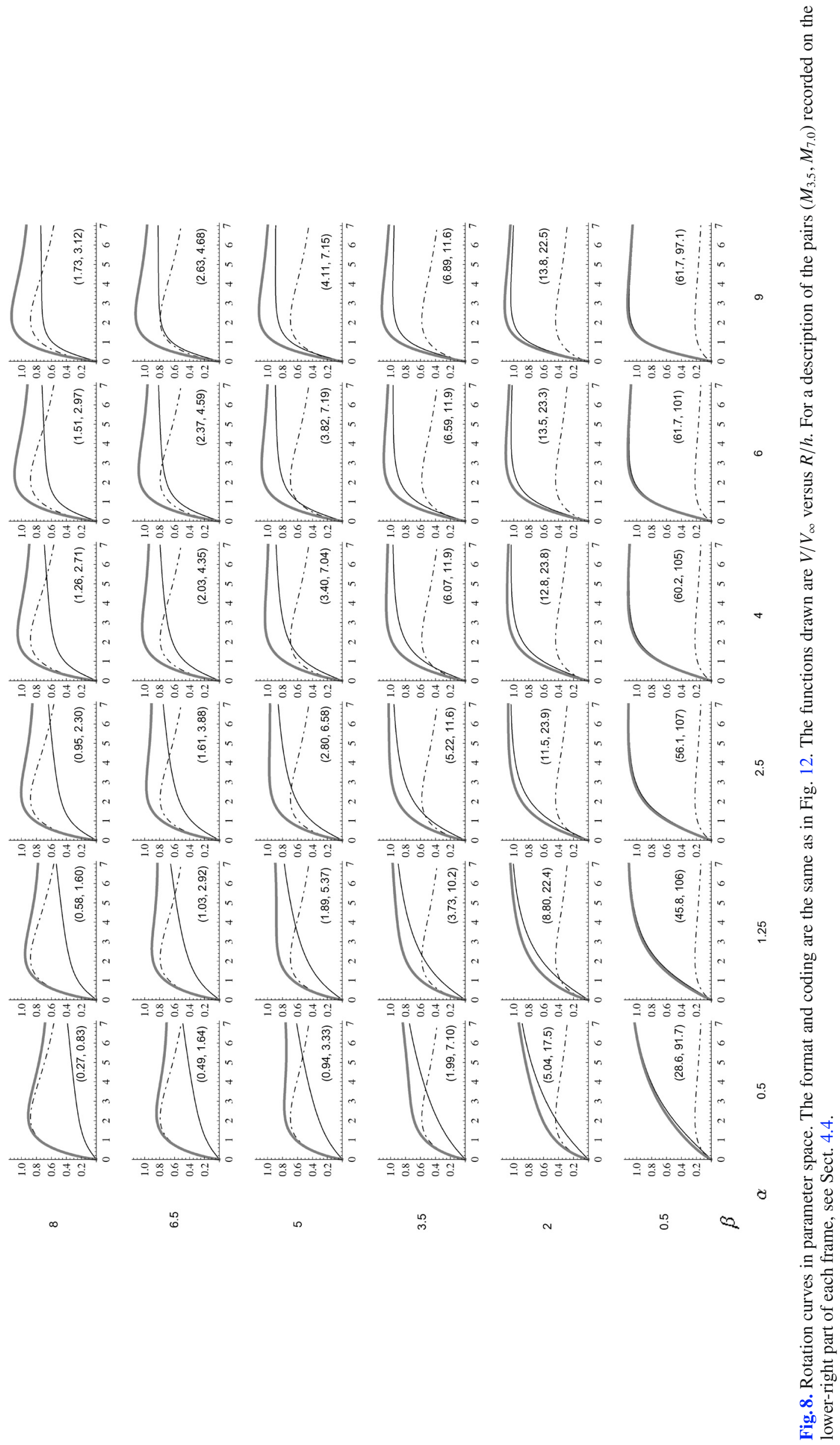




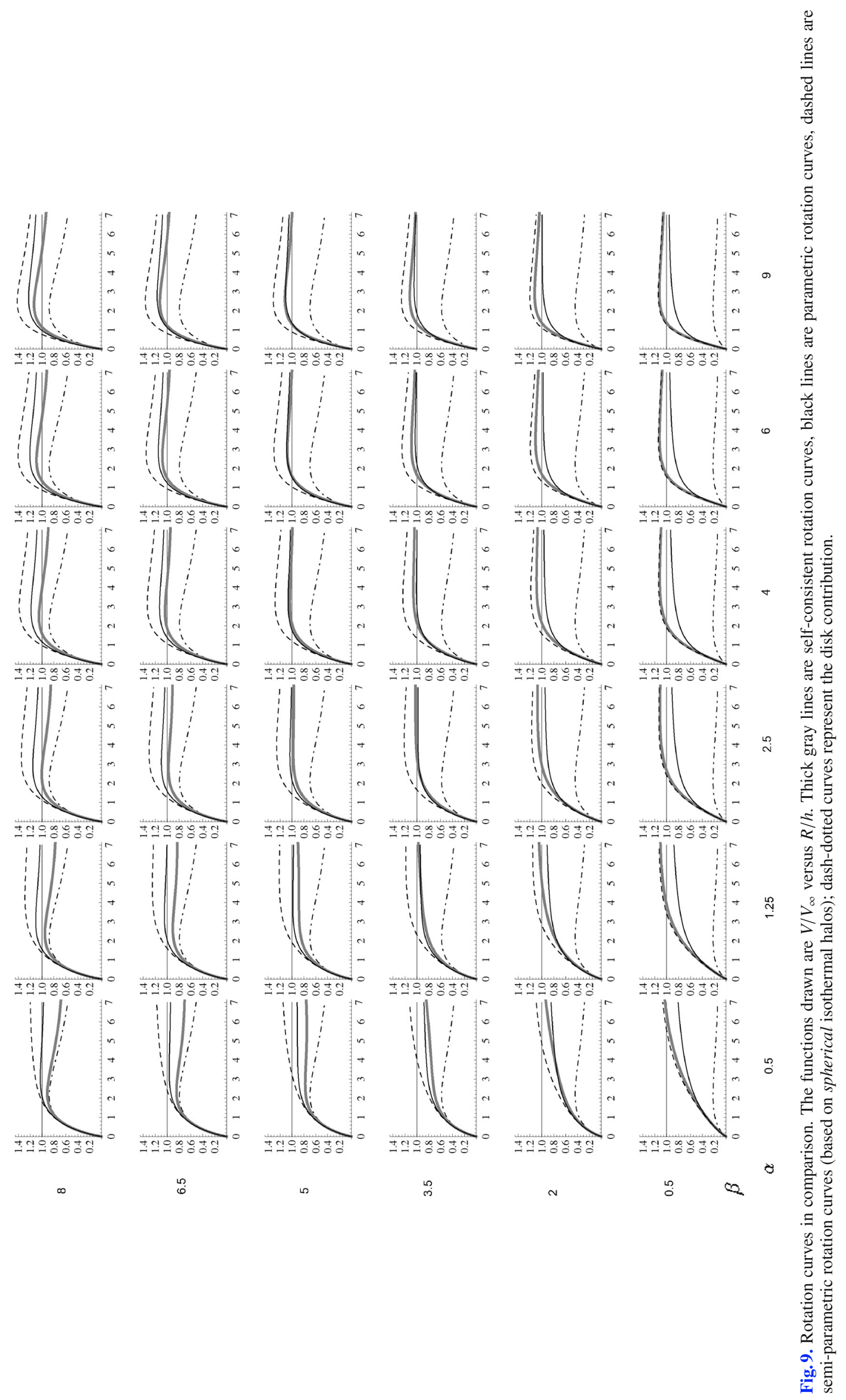


semi-parametric models (dashed lines) are shown here in comparison, in a general format and layout similar to those of Fig. 8. In each frame, the stellar disk contribution is given by the dashdotted line, while the constant line $V_{\text {mod }}=V_{\infty}$ is drawn to make the comparison easier. What clearly catches the eye is the remarkable uniformity of the parametric rotation curves, when compared to those of the semi-parametric models and of the self-consistent models. From a physical point of view, this uniformity is not justified, because it confirms that the parameterization used flattens out arbitrarily the properties of the physical isothermal solutions that it is meant to represent approximately.

\subsection{Conspiracy and degeneracy}

Figure 7 demonstrates that the available parameter space cannot accommodate values of $R_{\Omega} / h$ significantly different from unity, especially if we also require that the rotation curve be flat at intermediate radii. If galaxies were found with $R_{\Omega}$ too small or too large with respect to $h$, there would be no way for the picture of disks embedded in quasi-isothermal halos to hold and then one should be led to look for dark matter distributions significantly different from those characteristic of isothermal halos. This point will be further addressed in Appendix B, where the difficulty of fitting such "anomalous" rotation curve is demonstrated. Fortunately, the observed correlation Eq. (4) shows that the picture of a quasi-isothermal halo is indeed viable.

Figure 8 confirms that self-consistent models lead to rotation curves that are realistic, in the sense that they are smooth and featureless for a wide range of disk-to-halo mass ratios; note that the maximum disk solution associated with the correlation (4) (see Sect. 5.1 for description) is expected to occur at $\beta \approx 5$ (cf. Eq. (28)), approximately in the middle of the parameter range discussed and explored in this section. An inspection of Fig. 9 shows that parametric models are also characterized by this property, actually in an even more prominent way. In a sense, these findings demonstrate that we are not facing an issue of conspiracy or, at least, that the conspiracy is not as dramatic as often stated. In fact, differently from what is naturally expected and is often reported, no fine tuning is actually required in this problem, because for very different values of the halo-to-disk mass ratio the rotation curves resulting from the combination of a disk with a quasi-isothermal halo, turn out to be (surprisingly) smooth and flat. In this respect, the correlation represented by Eq. (4) implies a much tighter coupling between dark and luminous components in the $(\alpha, \beta)$ plane.

In the context of parametric models, these conclusions appear to be quite artificial. In fact, the use of the simple model associated with Eq. (2) removes a priori significant features that are characteristic of the regular isothermal sphere. Such non-natural uniformity in parameter space ends up in generating the problem of disk-halo degeneracy. In fact, the diagonal trend of the parametric models exhibited in the upper-left frame of Fig. 6 and in Fig. 7 reinforces the diagonal trend exhibited by the same models in Fig. 10, thus anticipating a problem of degeneracy of solutions along diagonal lines in parameter space.

The self-consistent models, instead, have a distinctly different behavior (see Figs. 5 and 7); for them, maximal flatness occurs in the vicinity of a single point of parameter space. We are then brought to conclude that degeneracy may be broken by selfconsistency. This last point is confirmed and better quantified in the following separate section.

\section{Self-consistent disk-halo decomposition: the removal of the disk-halo degeneracy}

\subsection{A preliminary test on a fiducial rotation curve}

As anticipated briefly in Sect. 2.1, the empirical correlation (4) found between the two relevant scale lengths $h$ and $R_{\Omega}$ allows us to make a preliminary test by focusing on a single, representative idealized case. Therefore, a discussion of specific observed objects, such as NGC 3198, is postponed and will be carried out separately (see following Sect. 5.2)

As a typical reference case, we consider a galaxy characterized by a purely exponential stellar disk, $\hat{\Sigma}(R) \equiv \exp (-\xi)$, and by the following rotation curve

$V(\xi, \tau) / V_{\infty}=1-\exp (-\xi / \tau)$.

If we take

$\tau \approx 1$

the correlation found in Eq. (4) is reproduced.

The simple analytical form of the adopted rotation curve (25) allows us to calculate explicitly the value of the "observed" parameter $\alpha_{\text {obs }}$ defined in Eq. (A.13):

$\alpha_{\text {obs }}=\alpha_{\text {obs }}(\tau)=\exp (2 \gamma) / 2 \tau^{2}$,

where $\gamma \approx 0.577$ denotes the Euler gamma constant, thus $\alpha_{\text {obs }}(\tau=1) \approx 1.59$; the following discussion will be based on the use of this value of $\alpha_{\text {obs }}$.

Note also that, for a given value of $\tau$, the rotation curve (25) will admit a well-defined maximum-disk decomposition. In practice, before carrying out any detailed decomposition, we may introduce, as a simple definition of maximum-disk, the disk characterized by the value of the dimensionless weight $\beta_{\max }(\tau)$ that gives, without any dark matter contribution, the best fit to the inner rotation curve in the interval $\left[0, R_{\Omega}\right]$. Note that this maximum-disk decomposition is designed in such a way to recover the central gradient of the rotation curve. Sometimes a different definition is used, by referring to the stellar disk which reaches the asymptotic rotation velocity in correspondence of the maximum of its rotation curve. This definition gives higher values of $\beta$ ( $\beta_{\max } \approx 10$ for an exponential disk), but it is completely independent of the properties of the central part of the rotation curve.

For $\tau$ given by Eq. (26), we find

$\beta_{\max }(1) \approx 5$

this value of $\beta$ identifies an important reference value to which the results obtained from the self-consistent decomposition will thus be compared. We recall that $\beta=5$ is approximately in the middle of the parameter range discussed and explored in Sect. 4.4.

The properties of the self-consistent models constructed in the present paper are best illustrated by describing their ability to fit the just described representative idealized case in comparison to a fit performed by more standard parametric analyses. For both methods the goodness of a disk-halo decomposition, defined by a pair $(\alpha, \beta)$, is quantified by the function

$\Xi(\alpha, \beta, \tau)=\frac{1}{V_{\infty}^{2}} \int_{0}^{7}\left[V_{\bmod }(\xi)-V(\xi, \tau)\right]^{2} \mathrm{~d} \xi$,

that is the integrated squared residuals between the "observed" rotation curve and the rotation curve calculated from the model 


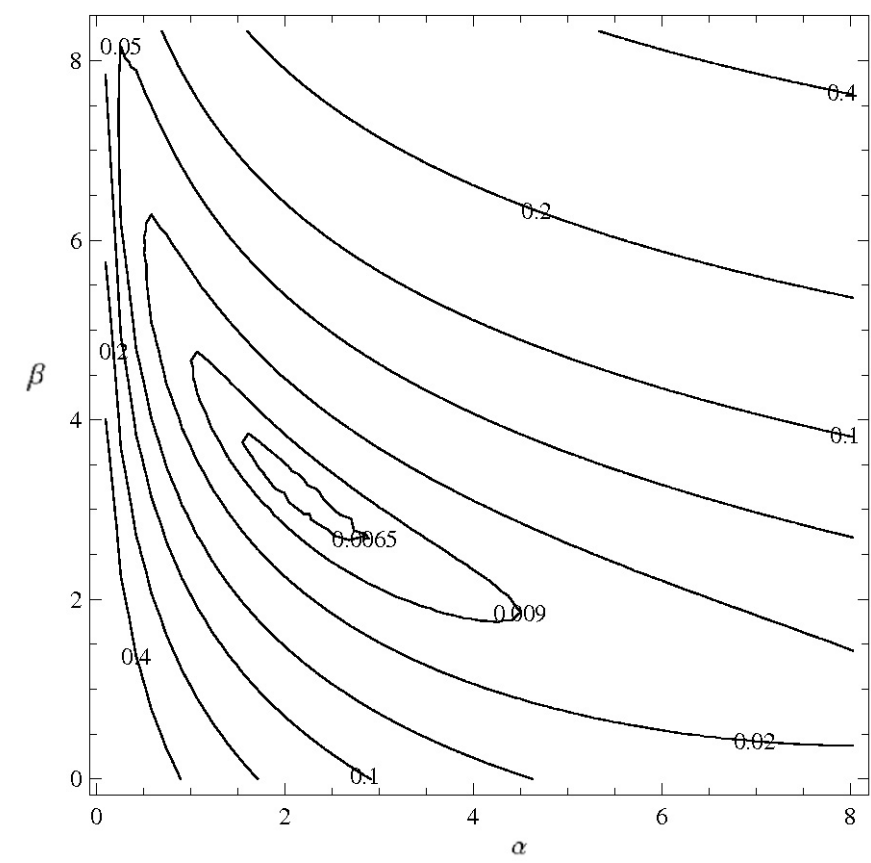

Fig. 10. Contours of the function $\Xi$ for the case of the parametric decomposition based on Eqs. (1, 2). As in similar plots (Figs. 11, B.1, B.2, B.4, B.5), the displayed contours are chosen arbitrarily to illustrate the properties of the $\Xi$ function.

$V_{\text {mod }}=\sqrt{V_{\mathrm{D}}^{2}+V_{\mathrm{DM}}^{2}}$. The cut of the integration at seven exponential lengths is a reasonable choice, consistent with the analysis of Sect. 4.2. In this preliminary test we do not treat the asymptotic velocity as an additional free parameter of the model. In other words, we just analyze the deviations defined by Eq. (29) in the $(\alpha, \beta)$ plane, at fixed $V_{\infty}$. The asymptotic velocity will be kept as a free parameter in Sect. 5.2, for the case of NGC 3198.

The results obtained from the standard parametric decomposition are shown in Fig. 10, which clearly exhibits the disk-halo degeneracy pattern. In particular, consider the contour marked by 0.02 . A sizable diagonal strip in parameter space consists of points that are basically equivalent from the point of view of the quality of the fit, even though they correspond to models that are physically very different, ranging from fairly light disks up to maximum-disk solutions (with $\beta$ changing by a factor higher than ten, from $\approx 0.5$ to $\approx 6.5$ ).

In contrast, Fig. 11 shows the contours of function (29) for the case in which the model rotation curve is calculated using the self-consistent method presented in this paper. The plot ranges and the values of the solid line contours plotted in Figs. 10 and 11 are the same. The self-consistent models give a better diskhalo decomposition for three distinct reasons: firstly, the value $\Xi_{\mathrm{bf}}=\Xi\left(\alpha_{\mathrm{bf}}, \beta_{\mathrm{bf}}, 1.02\right) \approx 0.00056$ of the function $\Xi$ corresponding to the best-fit model $\left(\alpha_{\mathrm{bf}}, \beta_{\mathrm{bf}}\right)$ is more than ten times smaller than the value attained by $\Xi$ based on the simple parametric decomposition $\left(\Xi_{\mathrm{bf}}^{\mathrm{nsc}} \approx 0.006\right)$; secondly, the area of the parameter space inside the contour $2 \Xi_{\mathrm{bf}} \approx 0.00112$ (or any other sensible multiple of $\left.\Xi_{\mathrm{bf}}\right)$ is quantitatively smaller, that is the bestfit model is identified more sharply; finally, the contours of the function $\Xi$ in the self-consistent case do not show any diagonal trend, thus confirming that the disk-halo degeneracy is removed.

In conclusion, the best-fit model identified by the selfconsistent analysis is defined by the following values of the relevant dimensionless parameters

$\left(\alpha_{\mathrm{bf}}, \beta_{\mathrm{bf}}\right) \approx(1.9,3.4)$.

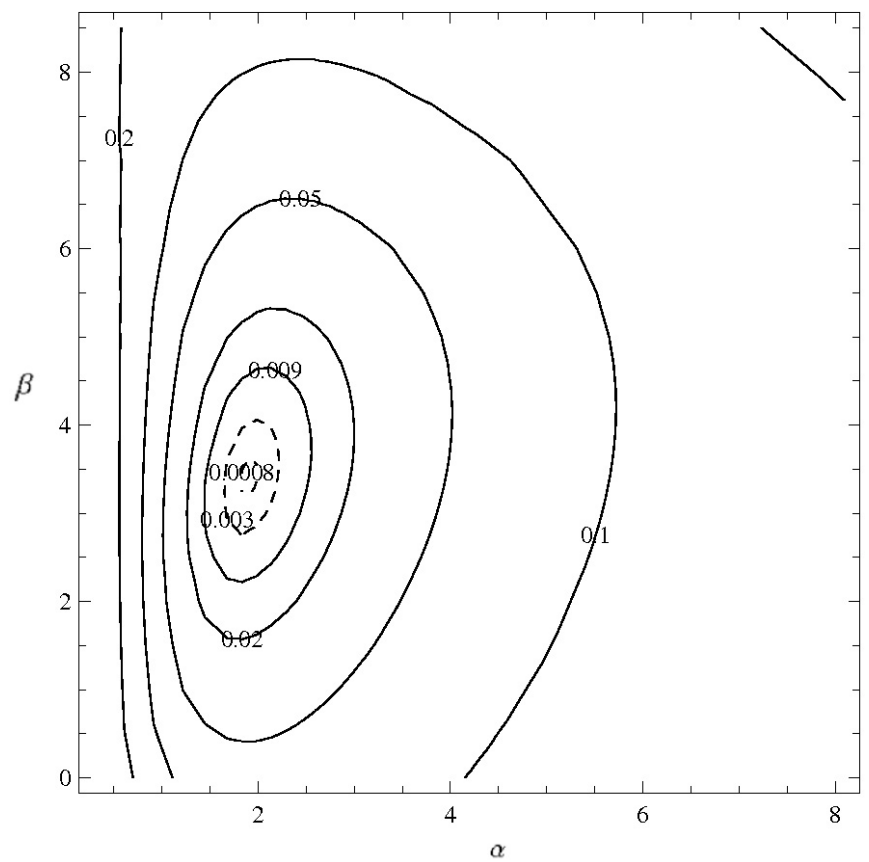

Fig. 11. Contours of the function $\Xi$ for the case of the self-consistent decomposition presented in this paper.

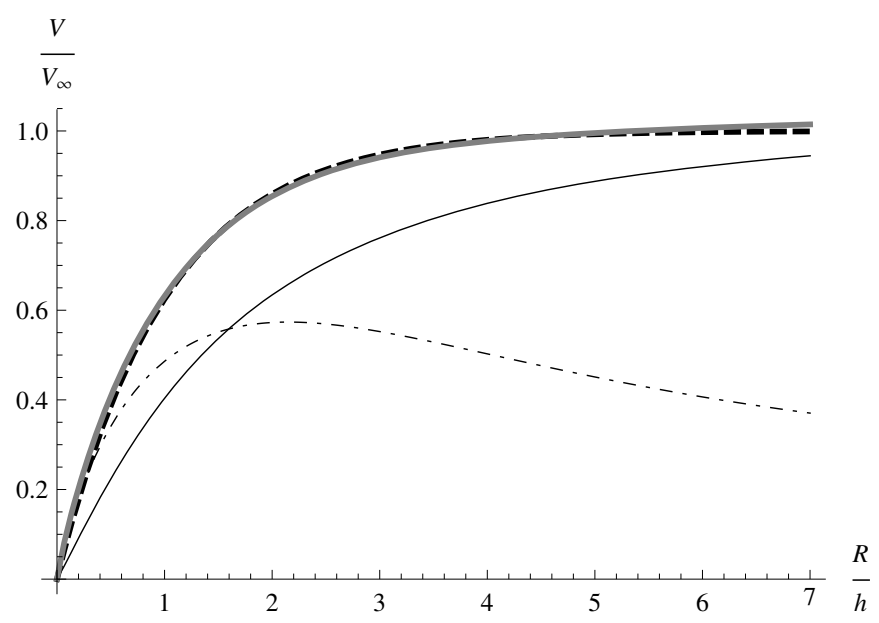

Fig. 12. Disk-halo decomposition associated with the best selfconsistent fit. The thick dashed line is the "observed" rotation curve, the thick solid line is the best-fit rotation curve, the thin lines represent the disk (dash-dotted) and the halo (solid) contributions.

Therefore, the best-fit model is significantly sub-maximal (see Eq. (28)). The associated rotation curve is shown in Fig. 12.

In order to better demonstrate the beneficial role of selfconsistency in breaking the disk-halo degeneracy, we now consider the self-consistent models that correspond to parameters with good (degenerate) parametric fits to the "observed" rotation curve, but are ruled out by the self-consistent analysis. In particular, as representative cases we choose the high-mass (i.e., high- $\beta$ ) and low-mass (i.e., low- $\beta$ ) ends of the $2 \Xi_{\mathrm{bf}}^{\mathrm{nsc}}$ contour in Fig. 10:

$$
\begin{aligned}
\left(\alpha_{\mathrm{hm}}, \beta_{\mathrm{hm}}\right) & \approx(0.9,5.4) \\
\left(\alpha_{\mathrm{lm}}, \beta_{\mathrm{lm}}\right) & \approx(6,1.75) .
\end{aligned}
$$

The self-consistent rotation curves associated with these two models are shown in Figs. 13 and 14. Note that the differences between the calculated total rotation curve and the 


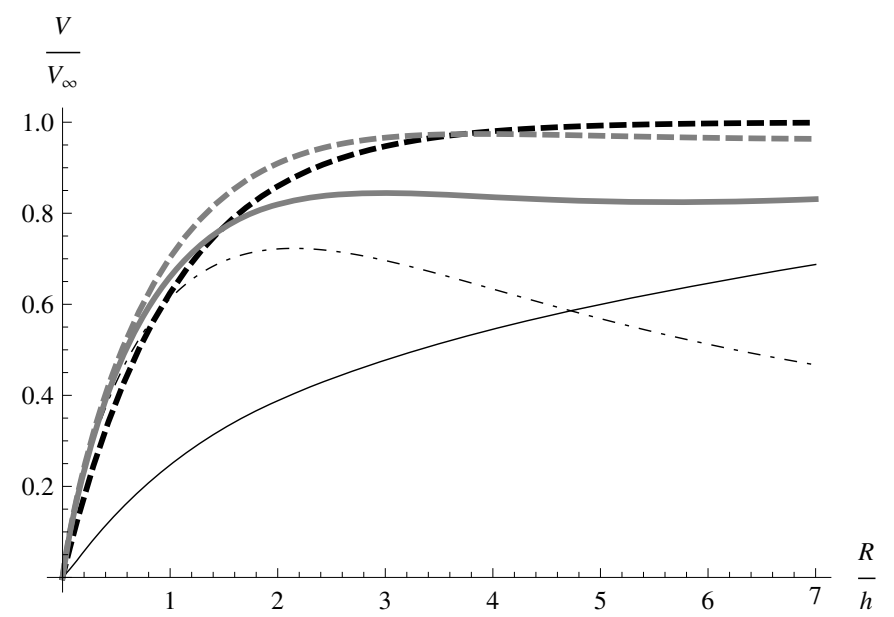

Fig. 13. Disk-halo decomposition by the $\left(\alpha_{\mathrm{hm}}, \beta_{\mathrm{hm}}\right)$ self-consistent model. The format and the notation are the same as in Fig. 12. The rotation curve of the corresponding $\left(\alpha_{\mathrm{hm}}, \beta_{\mathrm{hm}}\right)$ parametric model is displayed as a thick dashed gray line.

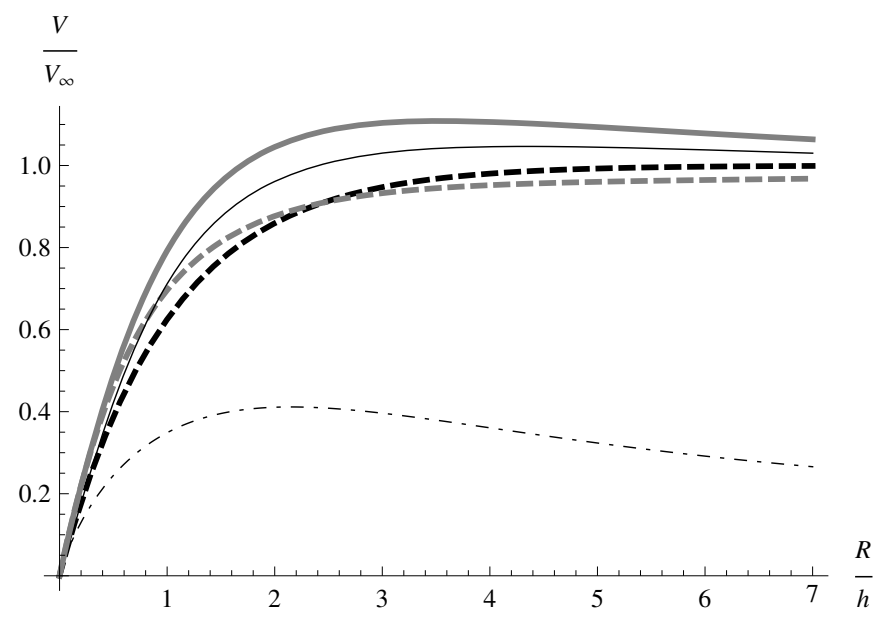

Fig. 14. Disk-halo decomposition by the $\left(\alpha_{\mathrm{lm}}, \beta_{\mathrm{lm}}\right)$ self-consistent model. The format and the notation are the same as in Fig. 12. The rotation curve of the corresponding $\left(\alpha_{\mathrm{lm}}, \beta_{\mathrm{lm}}\right)$ parametric model is displayed as a thick dashed gray line.

"observed" one are significantly larger than any realistic errorbar. Furthermore, the rotation curve of the high-mass case confirms the properties of the self-consistent rotation curves described in Sect. 4.4.

\subsection{Application to the case of NGC 3198}

As a specific example, we now consider the classical case of NGC 3198. We refer to the rotation curve reported in Table 2 of van Albada et al. (1985) and to their measure of the exponential length $(h=2.68 \mathrm{kpc})$. The value of the ratio $R_{\Omega} / h$ estimated from the rotation curve is $R_{\Omega} / h=0.6 \pm 0.1$. This is on the low side of the correlation reported in Sect. 2.1, that is the rotation curve of NGC 3198 rises more rapidly than average in its central parts. This feature may be partly related to the fact that the photometric profile of NGC 3198 is not exactly exponential in the innermost parts of the disk (see Begeman 1989; see also the discussion by Dutton et al. 2005). For simplicity, in the following we will consider the galaxy as bulgeless and we will ignore the contribution of the gaseous disk. We recall that the simple analysis provided in Sect. 5.1, applied to the inner parts of the rotation curve, suggests that the maximum-disk decomposition for NGC 3198 has $\beta_{\max }^{\mathrm{NGC}} 3198 \approx 10$.

In this case the rotation curve is provided with its observational errors. To evaluate quantitatively the goodness of a given disk-halo decomposition, we then adopt the classical likelihood function:

$\Lambda\left(V_{\infty}, \alpha, \beta\right)=\exp \left\{-\frac{1}{2} \sum_{i=1}^{i=28} \frac{\left[V_{\bmod }\left(R_{i}\right)-V\left(R_{i}\right)\right]^{2}}{\operatorname{Var}\left[V\left(R_{i}\right)\right]}\right\}$

where $\operatorname{Var}\left[V\left(R_{i}\right)\right]$ denotes the square of the error associated with the $i$ th rotation curve datapoint. In order to bring out the explicit dependence of the likelihood $\Lambda$ on the assumed value of $V_{\infty}$, we note that the previous equation can be written as:

$\Lambda\left(V_{\infty}, \alpha, \beta\right)=\exp \left\{-\frac{1}{2} \sum_{i=1}^{i=28} \frac{\left[V_{\infty} \cdot v_{\mathrm{mod}}\left(R_{i}, \alpha, \beta\right)-V\left(R_{i}\right)\right]^{2}}{\operatorname{Var}\left[V\left(R_{i}\right)\right]}\right\}$

in which the function $v_{\bmod }(R, \alpha, \beta)$ is normalized to unity at large radii.

We note that the rotation curve data from four to eleven exponential lengths suggest

$\hat{V}_{\infty}=(149.8 \pm 0.5) \mathrm{km} \mathrm{s}^{-1}$.

However, in order to avoid a possible bias that might be introduced by "freezing" this quantity, we treat the asymptotic velocity $V_{\infty}$ as a free parameter, to be determined by the fitting procedure together with $\alpha$ and $\beta$.

Figure 15 shows the results of the parametric disk-halo decomposition in the $(\alpha, \beta)$ plane, that is, after integrating out the dependence on $V_{\infty}$ in the likelihood Eq. (32):

$\hat{\Lambda}(\alpha, \beta)=\int_{0}^{\infty} \mathrm{d} V_{\infty} \Lambda\left(V_{\infty}, \alpha, \beta\right)$.

The contours identify regions of $68 \%$ and $95 \%$ confidence level; the best-fit model is marked by a full dot. Interestingly, two maxima exist, located along the disk-halo degeneracy strip described in the previous subsection, but with significantly different physical properties. In fact, for varying values of the asymptotic velocity $V_{\infty}$, the location of the best-fit model in the $(\alpha, \beta)$ plane (at fixed $V_{\infty}$ ) changes. As a result, the integrated likelihood Eq. (35) actually increases the amount of degeneracy associated with the parametric disk-halo decomposition. This is particularly evident from Fig. 16, which shows the projections along the two separate parameters $\alpha$ and $\beta$ of the the likelihood itself, exhibiting a clear bimodality. The disk-halo decomposition is unable to select between a solution very close to the maximum-disk and a decomposition with a much lighter disk. The corresponding decompositions are illustrated in Figs. 17 and 18, which show the two best-fit parametric decompositions, with projections on the $(\alpha, \beta)$ plane contained respectively in the two separate areas of the confidence regions in Fig. 15. This curious result may be related to the specific structure of the rotation curve of NGC 3198 (but see also Appendix B). In any case, the general statement holds, that the parametric decomposition is unable to lead to a unique determination of the weight of the stellar disk.

For completeness, we record the coordinates of the best-fit model in the $\left(V_{\infty}, \alpha, \beta\right)$ space, together with the related reduced $\chi^{2}$ value:

$(145.9,8.7,3.85,0.6)$. 


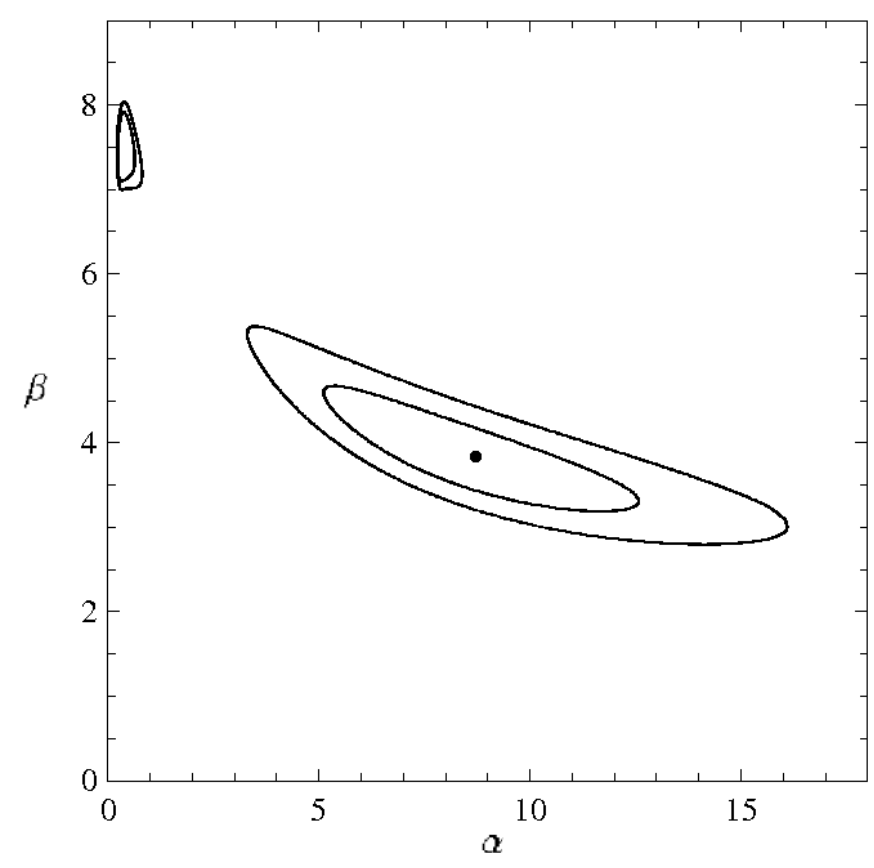

Fig. 15. Contours of $68 \%$ and $95 \%$ confidence regions in the $(\alpha, \beta)$ plane according to the likelihood defined in Eq. (35) for the parametric decomposition. The full dot marks the best-fit model.
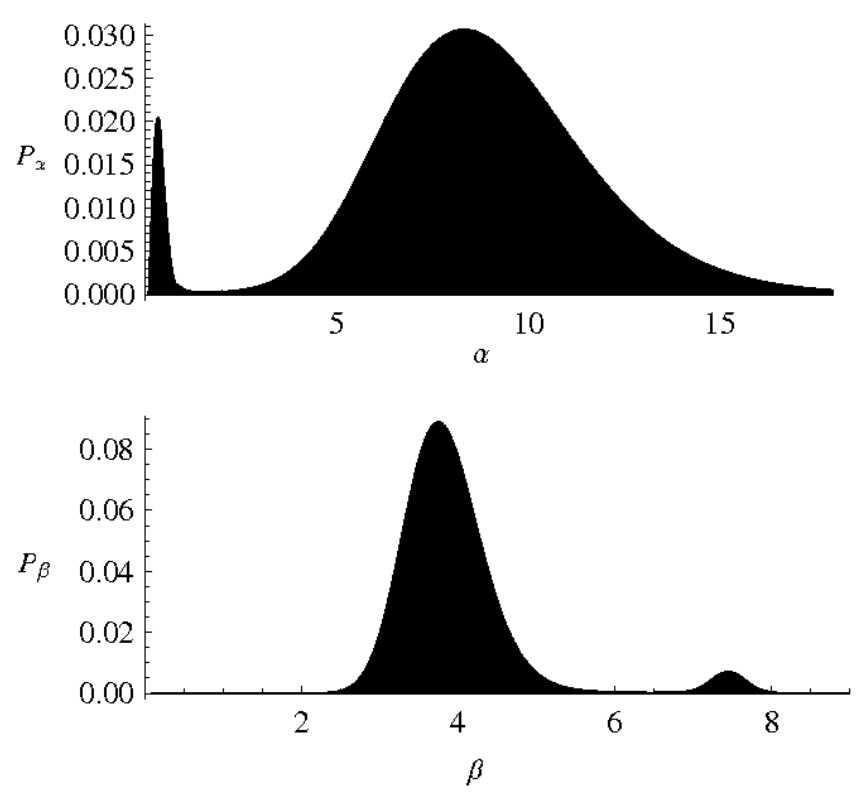

Fig. 16. Projections of the likelihood (32) for the single parameters $(\alpha, \beta)$ for the parametric decomposition.

Here we have defined

$\chi_{\text {red }}^{2}=\frac{1}{28-3} \sum_{i=1}^{i=28} \frac{\left[V_{\text {mod }}\left(R_{i}\right)-V\left(R_{i}\right)\right]^{2}}{\operatorname{Var}\left[V\left(R_{i}\right)\right]}$.

The situation is completely different if the disk-halo decomposition is performed by means of the self-consistent models presented in this paper. Figure 19 shows the contours of the $68 \%$ and $95 \%$ confidence levels and the best-fit model, calculated using the same likelihood as for the parametric case; note the significant change in their size compared to Fig. 15. At fixed values of the asymptotic velocity $V_{\infty}$, the general behavior of the best-fit models is similar to the one noted for the parametric analysis: the

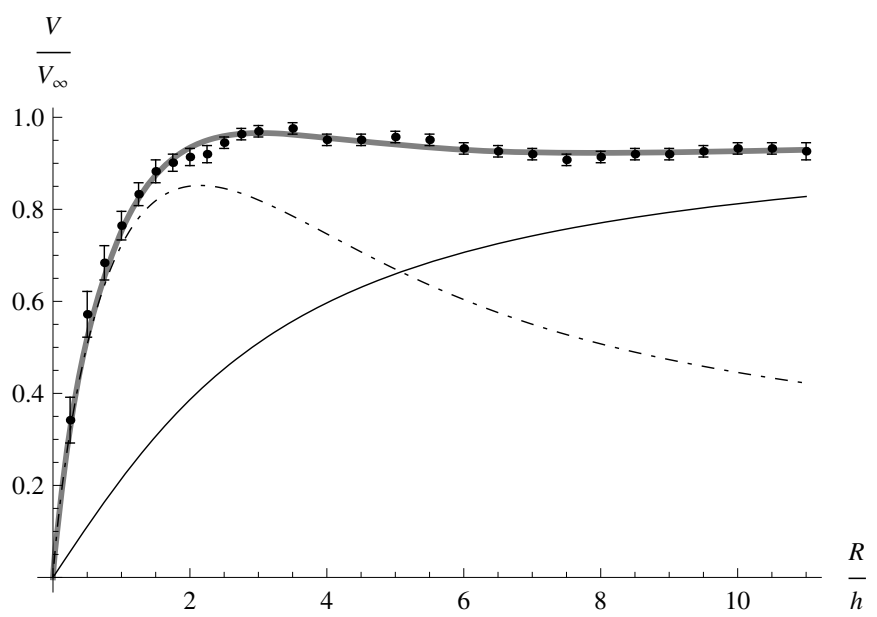

Fig. 17. Parametric disk-halo decomposition of NGC 3198 corresponding to the maximum of the likelihood in Fig. 15, with higher disk importance. The coding is the same as in Fig. 12.

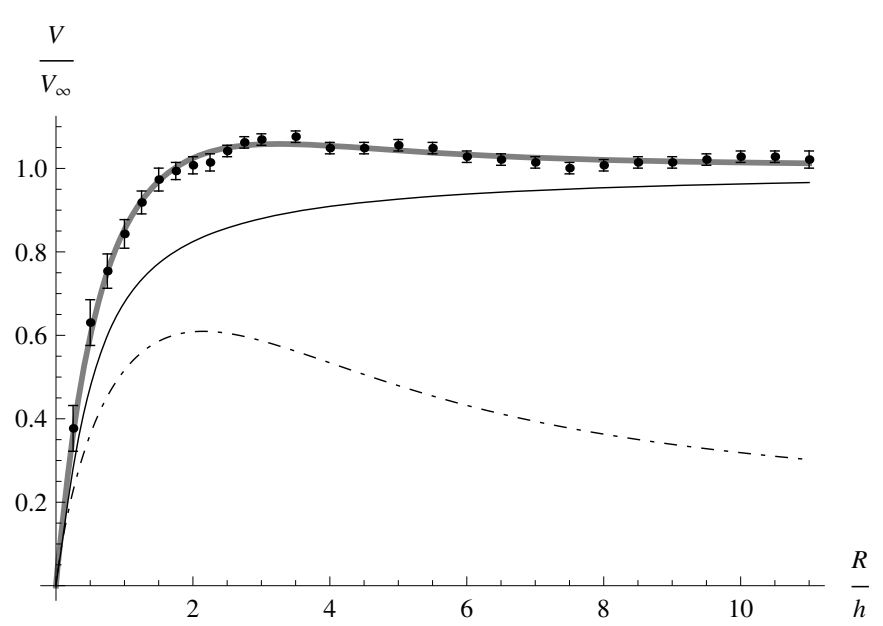

Fig. 18. Parametric disk-halo decomposition of NGC 3198 corresponding to the maximum of the likelihood in Fig. 15, with lower disk importance. The coding is the same as in Fig. 12.

higher the adopted value of $V_{\infty}$, the higher the value of $\beta$ found in the best-fit decomposition. However, here the differences cancel out in the integration Eq. (35), because of the different sharpness of the best-fits obtained at fixed values of $V_{\infty}$. As a result, the self-consistent decomposition is determined uniquely. In Fig. 20 the likelihood projections are indeed smooth and do not exhibit a bimodal shape. As for the parametric decomposition, we list the coordinates of the best-fit model in the $\left(V_{\infty}, \alpha, \beta\right)$ space, together with the related reduced $\chi^{2}$ value:

$(160.1,2.6,5.9,0.7)$.

The best-fit value of the asymptotic velocity $V_{\infty}$ is higher than the value suggested by the rotation curve in Eq. (34), consistent with the properties of the rotation curves displayed in Fig. 8 and with the suppression effect described in Sect. 4.4.

We note that the reduced $\chi^{2}$ value of the best self-consistent fits is somewhat higher than for the corresponding parametric fits. However, this difference is small and the reported values for the reduced $\chi^{2}$ account for globally good fits. This is evident from Fig. 21, which shows the best-fit self-consistent diskhalo decomposition together with the observed rotation curve. However, the model has a certain difficulty in fitting the second and third datapoints, which remain higher than the best-fit 
N. C. Amorisco and G. Bertin: Self-consistent nonspherical isothermal halos embedding zero-thickness disks

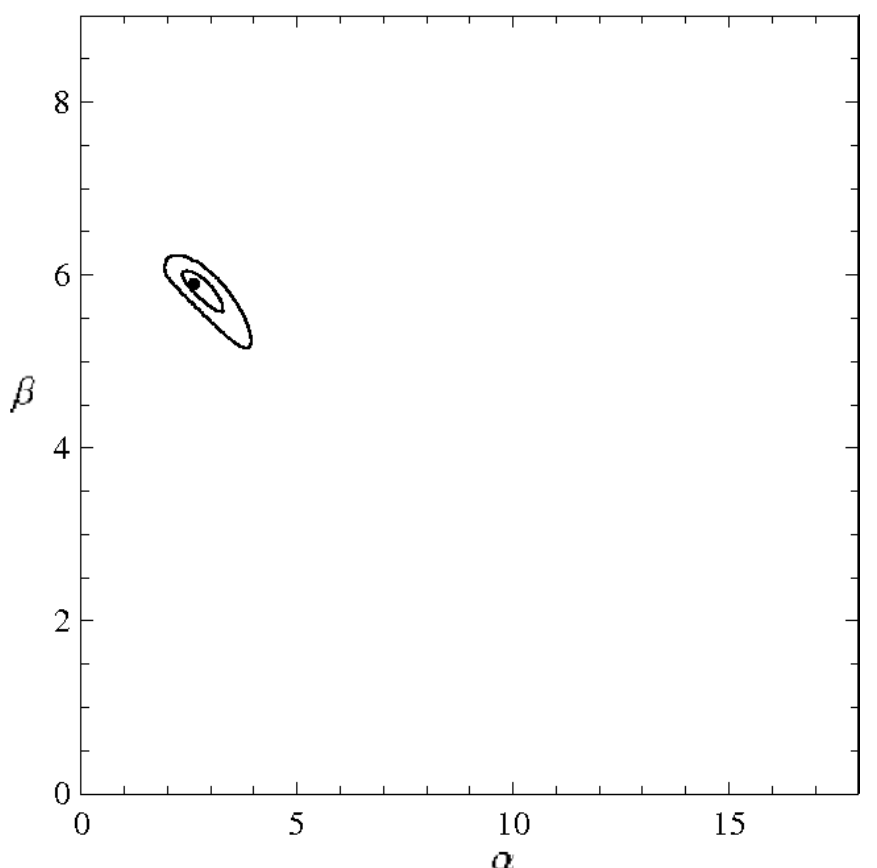

Fig. 19. Contours of $68 \%$ and $95 \%$ confidence regions in the $(\alpha, \beta)$ plane according to the likelihood defined in Eq. (32) for the selfconsistent decomposition. The full dot marks the best-fit model.
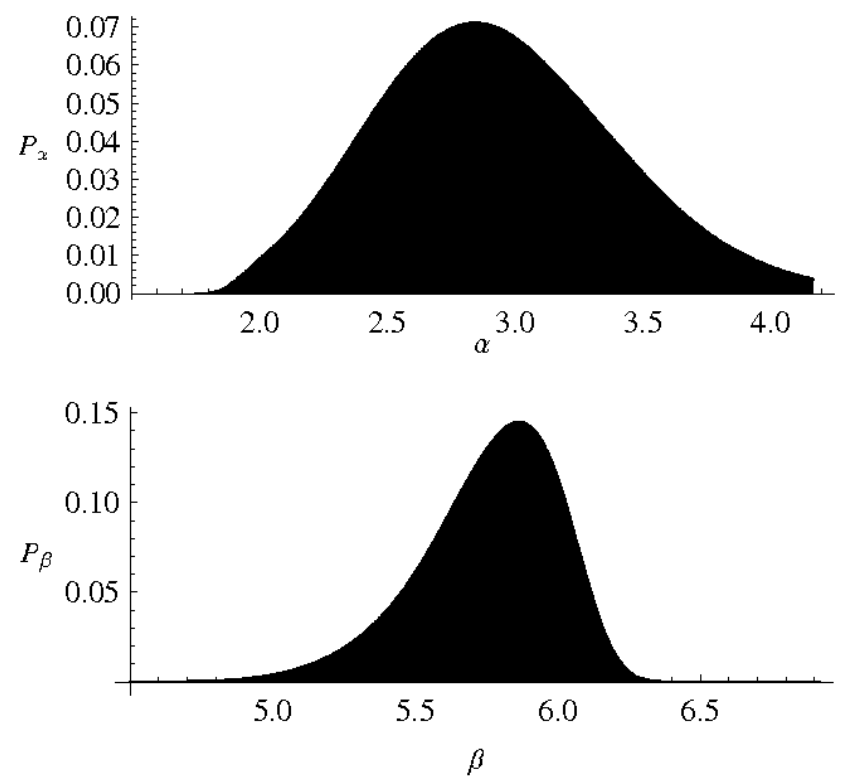

Fig. 20. Projections of the likelihood (32) for the single parameters $(\alpha, \beta)$ for the self-consistent decomposition.

self-consistent rotation curve. Clearly, this could be ascribed to the exponential modeling of the density profile of the visible matter, which, as noticed before, does not take into account the small bulge and the gas present in NGC 3198. Since these two datapoints are those that define the value of the ratio $R_{\Omega} / h$ for NGC 3198, Fig. 7 confirms that the best-fit self-consistent model has a slightly higher value for this ratio. Note also that the properties of the best-fit self-consistent decomposition are quite different from those found in the parametric analysis, with the bestfit model characterized by a disk of intermediate weight. As is evident from Fig. 21, the disk contribution to the rotation curve is important in the inner parts of the system. However, with its

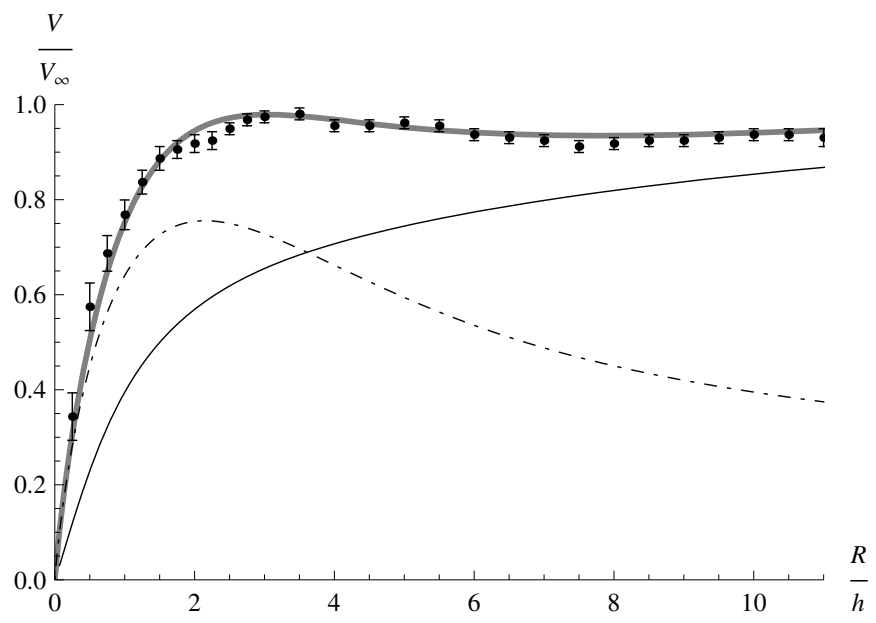

Fig. 21. Self-consistent disk-halo decomposition of NGC 3198. The coding is the same as in Fig. 12.

$\beta \approx 6$, the best-fit self-consistent model remains below the classical maximum-disk solutions that van Albada et al. (1985), for example, place at $\beta \gtrsim 9$.

\section{Conclusions}

This paper has addressed the construction of self-consistent models for a dark matter distribution assumed to be isothermal, in the presence of a zero-thickness disk. The models have then been applied to interpret the properties of observed rotation curves, with special attention to the issues of conspiracy and degeneracy that are known to affect their disk-halo decomposition.

We argue that what has been learned from the study of the strictly isothermal case should be applicable to the case of dark matter distributions characterized by a quasi-isothermal inner region (as is the case for the results of collisionless violent relaxation).

It would be interesting to test the results on the dark matter distributions predicted by current cosmological models, which definitely favor a density profile asymptotic to $r^{-3}$. This test could be performed with an approach similar to the one adopted in this paper, provided that we had a physically justified distribution function to start from. In particular, it would be interesting to test to what extent the cosmologically predicted distributions could be reconciled with the existence of cases, such as that of NGC 3198, in which the rotation curve remains very flat in a wide radial range.

Note that this paper has considered the impact of a given visible matter distribution on the distribution of dark matter. A study of the dynamical role of the dark halo in shaping the distribution of the visible matter would require considering a formation scenario that we do not wish to address here.

The main results obtained in this paper are the following:

- As a preliminary empirical study, we have shown that for both high surface brightness and low surface brightness disk galaxies the scale length $h$ of the light profile of pure disks correlates with the scale length $R_{\Omega}$ (defined in Eq. (3)) that characterizes the central growth of the rotation curve. This suggests that the distribution of dark and visible matter in pure disk galaxies is homologous, i.e. characterized by similar global relations in different galaxies, even if significantly different in size and type (HSB vs. LSB galaxies). Based on 
the results obtained in the rest of the paper, this point is interpreted as an indication of a gravitational interplay between luminous and dark components in the innermost parts of the system. We argue that this homology is more relevant and surprising as a conspiracy than the actual flatness of the observed rotation curves, because, especially in the context of parametric models, rotation curves turn out to be almost flat for very different values of the disk-to-halo mass ratio.

- We have constructed self-consistent models of isothermal halos embedding zero-thickness stellar disks, thus obtaining fully self-consistent axisymmetric isothermal dark matter halos. These make basically a two-parameter family of models; the two parameters measure the weight of the disk and the central density of the halo (see Eqs. (19), (16)). A third parameter, the asymptotic value of the rotation curve at large radii, is in principle free but can often be treated as a fixed parameter, given the fact that it is generally well constrained by the observations; note that a discussion with the third parameter kept free is given in our study of NGC 3198 (see fifth item in this subsection). The adopted solution procedure can easily incorporate the presence of a zero-thickness disk of gas (with desired surface density). In addition, a similar procedure could be followed to construct models based on different choices of the dark matter distribution function. In turn, we can also argue that the qualitative conclusions obtained in this paper by calculating the properties of strictly isothermal halos should also apply to more general systems where the halo distribution, in the region of interaction with the disk, is quasi-isothermal.

- We have shown and quantified that, for astrophysically realistic values of the two free parameters of the models, the dark matter halo is significantly flattened, especially in the regions where it coexists with the bright optical disk. Inside one exponential scale length, the dark matter distribution is that of a thick disk; nevertheless, its contribution to the rotation curve is approximately linear in the same region, denoting the presence of a natural core.

- The rotation curves of the self-consistent models have been shown to be generally smooth and flat in their intermediate regions. In particular, self-consistency has been proved to provide a physical "mechanism" able to flatten rotation curves in the region of the visible disk. This flattening effect is stronger in the region of parameter space that appears to represent realistic systems. This result goes against the naive expectation that self-consistency should tend to enhance features present in the potential well and then in the related rotation curve.

- We have shown that the application of self-consistent models to decompose an observed rotation curve breaks the diskhalo degeneracy. This result has been achieved under some simplifying assumptions. First, for simplicity we assumed that the density profile of the disk is a perfect exponential (although our self-consistent models can be constructed for any zero-thickness axysimmetric density profile for the stellar disk). Second the dark matter distribution function has been assumed to be a perfect isothermal; this point can be released in favor of other physically justified choices. The diskhalo degeneracy breaking has been obtained first by studying an idealized rotation curve representative of a typical rotation curve of a pure disk spiral galaxy and then by a direct application to the classical case of NGC 3198 . We have also provided arguments that support the idea that, to some extent, such degeneracy problem has been artificially created by the use of parametric models (such as the model associated with
Eq. (2)). In fact, the rotation curves generated by these parametric models have been shown to be exceedingly uniform and featureless.

- We have thus derived the best-fit self-consistent disk-halo decompositions and proved that they favor mass decompositions characterized by stellar disks of intermediate weight, which are still important in the central regions of the system, but are significantly lighter than maximum-disk solutions. On the other hand, the homology property noted in the first item of these Conclusions would still make such submaximal solutions consistent with the small scatter of the Tully-Fisher (1977) relation. Of course, a full demonstration of this conclusion would require that we moved beyond the case of the fiducial rotation curve (see Sect. 5.1), by studying, in the way described in Sect. 5.2 for NGC 3198, a complete sample of spiral galaxies. But this would obviously go well beyond the objectives of the present paper.

- The resulting rotation curve has been interpreted as resulting from a suppression effect, characteristic of self-consistency. Self-consistency would affect a proposed maximum-disk decomposition by significantly reducing the dark halo contribution to the total rotation curve and thus leading to worse fits, especially at intermediate radii.

- The resulting $M / L$ values for the stellar disk should be studied for each individual galaxy, in view of their implications and consistency with respect to what is known about the relevant stellar populations. In this context, an important test would be the study of LSB galaxies. However, this very important issue goes well beyond the goals of the present paper.

In a follow-up paper we will generalize the results of this paper to the case in which the disk is characterized by finite thickness, with results that may be of interest to projects such as the Disk Mass Project (Verheijen et al. 2004; Bershady et al. 2010a,b).

Acknowledgements. We wish to thank Luca Ciotti, Wyn Evans, Renzo Sancisi, Tjeerd van Albada and an anonymous Referee for making a number of interesting comments and suggestions.

\section{References}

Barnabé, M., Czoske, O., Koopmans, L. V. E., et al. 2009, MNRAS, 399, 21 Bershady, M. A., Verheijen, M. A. W., Swaters, R. A., et al. 2010a, ApJ, 716, 198

Bershady, M. A., Verheijen, M. A. W., Westfall, K. B., et al. 2010b, ApJ, 716, 234

Begeman, K. G. 1989, A\&A, 223, 47

Bertin, G. 1993, in Structure, dynamics and chemical evolution of elliptical galaxies, ed. I. J. Danziger, W. W. Zeilinger, \& K. Kjaer, ESO Conference and Workshop Proceedings, 45, 243

Bertin, G. 2000, Dynamics of Galaxies (Cambridge University Press)

Bertin, G., \& Lin, C. C. 1996, Spiral structure in galaxies: a density wave theory (Cambridge: MA MIT Press)

Bertin, G., \& Mark, J. W.-K. 1980, A\&A, 88, 289

Bertin, G., \& Varri, A. 2008, ApJ, 689, 1005

Bertin, G., Saglia, R. P., \& Stiavelli M. 1992, ApJ, 384, 423

Casertano, S., \& van Gorkom, J. H. 1991, AJ, 101, 1231

de Blok, W. J. G., van der Hulst, J. M., \& Bothun, G. D. 1995, MNRAS, 274, 235

de Blok, W. J. G., \& McGaugh, S. S. 1997, MNRAS, 290, 533

Dutton, A. A., Courteau, S., de Jong, R., \& Carignan, C. 2005, ApJ, 619, 218

Franx, M., van Gorkom, J. H., \& de Zeeuw, T. 1994, ApJ, 436, 642

Freeman, K. C. 1970, ApJ, 160, 811

Gerhard, O., Kronawitter, A., Saglia, R. P., \& Bender, R. 2001, AJ, 121, 1936

Gnedin, O. Y., Brown, W. R., Geller, M. J., \& Kenyon, S. J. 2010, ApJ, 720, L108

Kalnajs, A. 1983, in Internal kinematics and dynamics of galaxies, ed. E. Athanassoula (Dordrecht, The Netherlands: Reidel), 87

Kautsch, S. J. 2009, Publ. Astron. Soc. Pacif., 121, 1297

Kent, S. M. 1986, AJ, 91, 1301 
N. C. Amorisco and G. Bertin: Self-consistent nonspherical isothermal halos embedding zero-thickness disks

McGaugh, S. S., \& Bothun G. D. 1994, AJ, 107, 530

O’Brien, J. C., Freeman, K. C., van der Kruit, P. C., Bosma, A. 2010, A\&A, 515, A60

Olling, R. P., \& Merrifield, M. R. 2000, MNRAS, 311, 361

Ostriker, J. P., \& Peebles, P. J. E. 1973, ApJ, 186, 467

Prendergast, K. H., \& Tomer, E. 1970, ApJ, 75, 674

Roberts, M. S. 1976, Comm. Astrophys., 6, 105

Saglia, R. P., Bertin, G., \& Stiavelli, M. 1992, ApJ, 384, 433

Sancisi, R. 2004, International Astronomical Union Symposium, 220, held 2125 July, 2003 in Sydney, Australia, ed. S. D. Ryder, D. J. Pisano, M. A. Walker, \& K. C. Freeman (San Francisco: ASP), 233

Sancisi, R., \& van Albada, T. S. 1987, Dark matter in the universe, Proc. IAU Symp., Princeton, NJ, June 24-28 (Dordrecht: D. Reidel Publishing Co.), 67 Swaters, R. A., Madore, B. F., \& Trewella, M. 2000, ApJ, 531, 107
Toomre, A. 1963, ApJ, 139, 385

Trott, C. M., Treu, T., Koopmans, L. V. E., \& Webster, R. L. 2010, MNRAS, 401,540

Tully, R. B., \& Fisher, J. R. 1977, A\&A, 54, 661

Tully, R. B., Verheijen, M. A. W., \& Pierce, M. J. 1996, AJ, 112, 2571

van Albada, T. S., \& Sancisi, R. 1986, Phil. Trans. R. Soc. Lond. A, 320, 447

van Albada, T. S., Bahcall, J. N., Begeman, K. G., \& Sancisi, R. 1985, ApJ, 295, 305

van de Hulst, H. C., Raimond, E., \& van Woerden, H. 1957, Bull. Astron. Inst. Neth., 14, 1

Verheijen, M. A. W., \& Sancisi, R. 2001, A\&A, 370, 765

Verheijen, M. A. W., Bershady, M. A., Andersen, D. R., et al. 2004, Astron. Nachr., 325, 151

Widrow, L. M., Pym, B., \& Dubinski, J. 2008, ApJ, 679, 1239

Pages 18 to 20 are available in the electronic edition of the journal at http: //www . aanda. org 


\section{Appendix A: Construction of the models by an iterative procedure}

Equation (18), with the boundary conditions (20), is solved by means of a modified version of the iterative procedure presented earlier by Prendergast \& Tomer (1970) in view of a completely different problem (the study of nonspherical models for rotating elliptical galaxies). It is based on the classical expansion in Legendre polynomials, a natural approach in the case of axisymmetric systems. However, the original method is viable only for gravitational potentials that are regular at large radii. In contrast, in our model, the potential of the isothermal halo diverges at large radii. This difficulty has been overcome by separating the potential of the dark matter halo into two different parts: a regular part, which converges to finite values at large radii, and a divergent one, which follows the asymptotic prescription of Eq. (20). Below we provide the details of the technique developed in the present paper. Here it is convenient to work with standard spherical coordinates $(r, \phi, \theta)$ and their dimensionless analogue $(\eta, \phi, \theta)$. We start by recalling briefly the method devised by Prendergast \& Tomer (1970). The solution of the twodimensional Poisson equation

$\nabla^{2} \psi(\eta, \theta)=\hat{\rho}[\eta, \theta ; \psi(\eta, \theta)]$

is obtained by iteratively solving the equations

$\nabla^{2} \psi^{(n+1)}(\eta, \theta)=\hat{\rho}\left[\eta, \theta ; \psi^{(n)}(\eta, \theta)\right]$

to which the same boundary conditions as in Eq. (A.1) are imposed. The potential at the iterative step $(n+1), \psi^{(n+1)}$, is obtained from the potential at the previous step, $\psi^{(n)}$, by solving Eq. (A.2) exactly, using the standard multipole expansion in Legendre polynomials, here denoted by $P_{k}$ :

$\hat{\rho}^{(n)}(\eta, \theta)=\sum_{k=0}^{\infty} \hat{\rho}_{k}^{(n)}(\eta) P_{k}(\cos \theta)$,

and

$\psi^{(n+1)}(\eta, \theta)=\sum_{k=0}^{\infty} \psi_{k}^{(n)}(\eta) P_{k}(\cos \theta)$

For completeness, we display the general solution of the $n$th iterative step:

$$
\begin{aligned}
\psi^{(n+1)}(\eta, \theta)= & \Psi+\left[\int_{0}^{\eta} \eta^{\prime} \hat{\rho}_{0}^{(n)}\left(\eta^{\prime}\right) \mathrm{d} \eta^{\prime}-\frac{1}{\eta} \int_{0}^{\eta} \eta^{\prime 2} \hat{\rho}_{0}^{(n)}\left(\eta^{\prime}\right) \mathrm{d} \eta^{\prime}\right] \\
& -\sum_{k=2}^{\infty} \frac{P_{k}(\cos \theta)}{2 k+1}\left[\eta^{k} \int_{\eta}^{\infty} \eta^{\prime-k} \hat{\rho}_{k}^{(n)}\left(\eta^{\prime}\right) d \eta^{\prime}\right. \\
& \left.+\frac{1}{\eta^{k+1}} \int_{0}^{\eta} \eta^{\prime k+2} \hat{\rho}_{k}^{(n)}\left(\eta^{\prime}\right) \mathrm{d} \eta^{\prime}\right]
\end{aligned}
$$

where we have used the notation $\psi(0, \theta)=\Psi$. The iteration can be stopped when the desired accuracy prescription is met, as for example when in the relevant domain $\mathcal{D}$ we find that

$$
\max _{(\xi, \zeta) \in \mathcal{D}}\left|\frac{\psi^{(n+1)}-\psi^{(n)}}{\psi^{(n+1)}+\psi^{(n)}}\right|<\epsilon .
$$

The dark matter gravitational potential is then separated into two parts:

$\psi_{\mathrm{DM}} \equiv \psi_{\mathrm{asy}}+\psi$

The potential defined as $\psi_{\text {asy }}$ obeys the Poisson equation

$\nabla^{2} \psi_{\text {asy }}=\hat{\rho}_{\text {asy }}$ and has the same asymptotic behavior as in Eq. (20). In this way the potential $\psi$ converges to zero at large radii and the related Poisson equation

$$
\left(\frac{1}{\xi} \frac{\partial}{\partial \xi} \xi \frac{\partial}{\partial \xi}+\frac{\partial^{2}}{\partial \zeta^{2}}\right) \psi=-\alpha \exp \left[\psi_{\text {asy }}+\psi_{\mathrm{D}}+\psi\right]-\hat{\rho}_{\text {asy }}
$$

can be directly solved by the iterative multipole expansion outlined previously.

Obviously, there is an infinite number of pairs $\left(\hat{\rho}_{\text {asy }}, \psi_{\text {asy }}\right)$ that meet the conditions of Eqs. (A.8) and (20). Therefore, the construction method proposed below just reflects one reasonable choice. We decided to take $\hat{\rho}_{\text {asy }}$ and $\psi_{\text {asy }}$ with spherical symmetry, because the relevant asymptotic condition for $\psi_{\text {asy }}$ is characterized by spherical symmetry and because the spherical Poisson equation admits a simple explicit solution:

$\psi_{\text {asy }}(\eta)=\psi_{\text {asy }}(0)+\int_{0}^{\eta} \eta^{\prime} \hat{\rho}_{\text {asy }}\left(\eta^{\prime}\right) \mathrm{d} \eta^{\prime}-\frac{1}{\eta} \int_{0}^{\eta} \eta^{\prime 2} \hat{\rho}_{\text {asy }}\left(\eta^{\prime}\right) \mathrm{d} \eta^{\prime}$

The precise choice of the form of the density $\hat{\rho}_{\text {asy }}$ is guided by the goal of simplifying the following numerical procedure to solve Eq. (A.9). Different choices of the density $\hat{\rho}_{\text {asy }}$ correspond to different shapes of the potential $\phi$ for the same pair of free parameters $(\alpha, \beta)$. The adopted practical recipe to construct the density $\hat{\rho}_{\text {asy }}$ turns out to be useful and efficient.

We start by defining what we call an "observed" pseudopotential, constructed from the observed rotation curve:

$\Phi_{\text {obs }}(r)=\int_{0}^{R} \frac{V^{2}(s)}{s} \mathrm{~d} s$.

At large radii it has the asymptotic expression

$\Phi_{\mathrm{obs}}(r) \sim V_{\infty}^{2} \ln \left(\frac{r}{r_{0}^{\mathrm{obs}}}\right)$,

where the radius $r_{0}^{\text {obs }}$ can be calculated from the precise form of the rotation curve. The behavior of Eq. (A.12) naturally relates the potential $\Phi_{\text {obs }}$ (and its dimensionless counterpart $\psi_{\text {obs }}$ ) to a density distribution with isothermal functional shape:

$\frac{1}{\eta^{2}} \frac{\mathrm{d}}{\mathrm{d} \eta} \eta^{2} \frac{\mathrm{d}}{\mathrm{d} \eta} \psi_{\mathrm{obs}}(\eta) \sim-\alpha_{\mathrm{obs}} \exp \left[\psi_{\mathrm{obs}}(\eta)\right] ; \eta \gg 1$

As shown in Eq. (10), the value of the constant $\alpha_{\text {obs }}$ is determined by the value of the radius $r_{0}^{\text {obs }}$ only, and is thus fixed. Having made this points clear, we can define $\hat{\rho}_{\text {asy }}$ to be

$\hat{\rho}_{\text {asy }}(\eta) \equiv-\alpha_{\text {obs }} \exp \left[\psi_{\text {obs }}\left(\sqrt{\eta^{2}+\eta_{\mathrm{c}}^{2}}\right)\right]+\hat{\rho}_{\mathrm{D}}^{(0)}(\eta)$,

where we have introduced a regularizing core structure with characteristic size $\eta_{c}$ and subtracted the monopole term of the stellar density

$\rho_{\mathrm{D}}^{(0)}(\eta) \equiv \frac{\beta}{2} \int_{-1}^{1} \hat{\Sigma}(\eta) \delta(\zeta) P_{0}(\cos \theta) \mathrm{d} \cos \theta=\frac{\beta}{2} \frac{\hat{\Sigma}(\eta)}{\eta}$.

With this density profile, from Eq. (A.10) we calculate the gravitational potential $\psi_{\text {asy }}$, using the free constant $\psi_{\text {asy }}(0)$ to make it meet exactly the asymptotic behavior required by Eq. (20). The determination of the correct value of the constant $\psi_{\text {asy }}(0)$ determines also the boundary condition to be used for Eq. (A.9), which is set by the prescription:

$\psi_{\mathrm{T}}(0,0)=\psi(0,0)+\psi_{\text {asy }}(0)+\psi_{\mathrm{D}}(0,0)=0$. 


\section{A.1. The truncation of the angular expansion}

A code has been written to solve Eq. (A.9) through the technique described in the previous section. The code is able to manage automatically the appropriate number of multipole terms to meet a required accuracy (see Eq. (A.6)). At each iterative step, the relevant number of multipole orders is calculated from the following prescription:

$\frac{\psi_{\bar{k}}^{(n)}(\bar{\eta}) P_{\bar{k}}(\cos \bar{\theta})}{\sum_{k=0}^{\bar{k}-1} \psi_{k}^{(n)}(\bar{\eta}) P_{k}(\cos \bar{\theta})}>10^{-1} \epsilon$.

In words, at the $n$th iteration step, the multipole term of order $\bar{k}$ is retained in the expansion if its (relative) contribution to the gravitational potential $\psi^{(n)}$ (calculated with the previous $\bar{k}-1$ multipole orders only) is comparable to the accuracy level $\epsilon$ we want to meet. This comparison is made at the coordinates $(\bar{\eta}, \bar{\theta})$, chosen in the region where the deviation from spherical symmetry is strongest: $\bar{\theta} \approx \pi / 2$ and $\bar{\eta} \approx 1 \div 2$.

The models described in the paper are calculated with an accuracy prescription of $\epsilon=10^{-4}$. Obviously, the number of iterations required by such precision depends on the gravitational importance of the stellar disk (which determines the flattening of the halo). For values of the pair $(\alpha, \beta)$ that represent an astrophysically realistic configuration (see Sect. 4), the number of required iterations is $10 \div 20$, with a number of multipole orders of $12 \div 20$.

\section{Appendix B: Anomalous rotation curves}

In this section we will analyze the case of rotation curves with inner gradients that are significantly different from the one identified by Eq. (4). These rotation curves represent systems with a "wrong tuning" between the disk and halo components, in the sense described in Sect. 2. We adopt the same simple parametrization for the rotation curve shape as in Eq. (25) and consider two different values for the parameter $\tau$, taken to be significantly far from the value which that reproduces the correlation in Eq. (4). On the fast rising end, we take $\tau_{\mathrm{f}}=0.4$ (i.e. $R_{\Omega} / h \approx 0.44$ ), while on the opposite side of a slowly rising rotation curve we take $\tau_{\mathrm{s}}=2.3$ (i.e. $R_{\Omega} / h \approx 2.53$ ). We apply the selfconsistent and the parametric disk-halo decomposition to these two anomalous rotation curves and refer to the same function $\Xi$ as in Eq. (29) to quantify the quality of the fit.

The results for the fast rising rotation curve are shown in Figs. B.1 and B.2. In the parametric decomposition method we find again the bimodality of the fit, as noted in the case of NGC 3198. The self-consistent method instead identifies a decomposition with an important stellar disk; the fit for the rotation curve is illustrated in Fig. B.3.

The results for the slowly rising rotation curve are shown in Figs. B.4 and B.5. The degeneracy pattern is clearly present in the parametric decomposition, but in this case it is less marked with respect to the cases studied earlier in this paper. Here, the parametric fit is "pushed" in the direction of an insignificant disk, because the observed $R_{\Omega}$ is too small and can only be ascribed to a dominant dark matter halo. On the other hand, the self-consistent decomposition appears to be able to handle also this case (see Fig. B.6).

From the point of view of the quality of the fits, it is apparent that both decomposition methods work best when fitting the case of $\tau \approx 1$, that is for a rotation curve that follows the empirical correlation illustrated in Fig. 1. This is the aspect of conspiracy that was introduced and described in Sect. 2.1. In this case, the

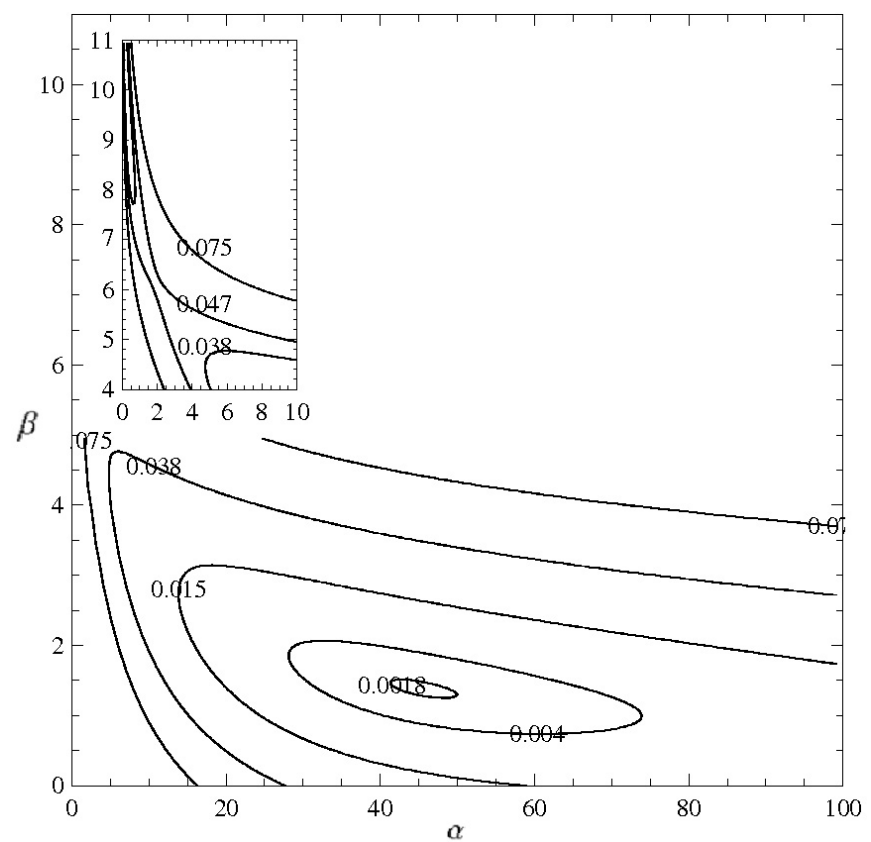

Fig. B.1. Contours of the function $\Xi$ for the parametric decomposition (based on Eqs. $(1,2)$ ) of the rotation curve defined by Eq. (25) with $\tau_{\mathrm{f}}=0.4$.

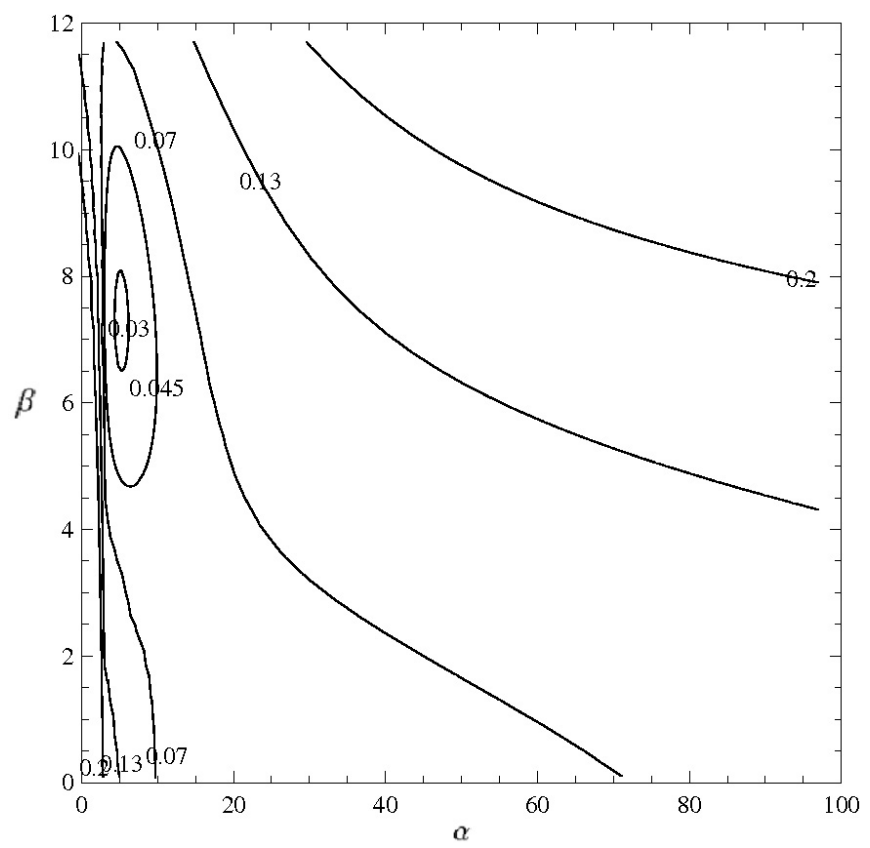

Fig. B.2. Contours of the function $\Xi$ for the self-consistent decomposition of the rotation curve defined by Eq. (25) with $\tau_{\mathrm{f}}=0.4$.

values of the function $\Xi$ corresponding to the best-fitting $(\alpha, \beta)$ pairs are the smallest. In particular, Fig. B. 3 shows that the case of a fast rising rotation curve cannot be properly described without including a central concentrated mass component, such as a bulge; the value of the residuals $\Xi$ in correspondence of the bestfit (see Fig. B.2) is considerably higher if compared for example to the good fit of Fig. 12. Similarly, for the opposite case of a slowly rising rotation curve, it is the parametric model which is quite unable to account for such a shape of the rotation velocity, with a similar high value for the best-fit residuals (see Fig. B.4). 


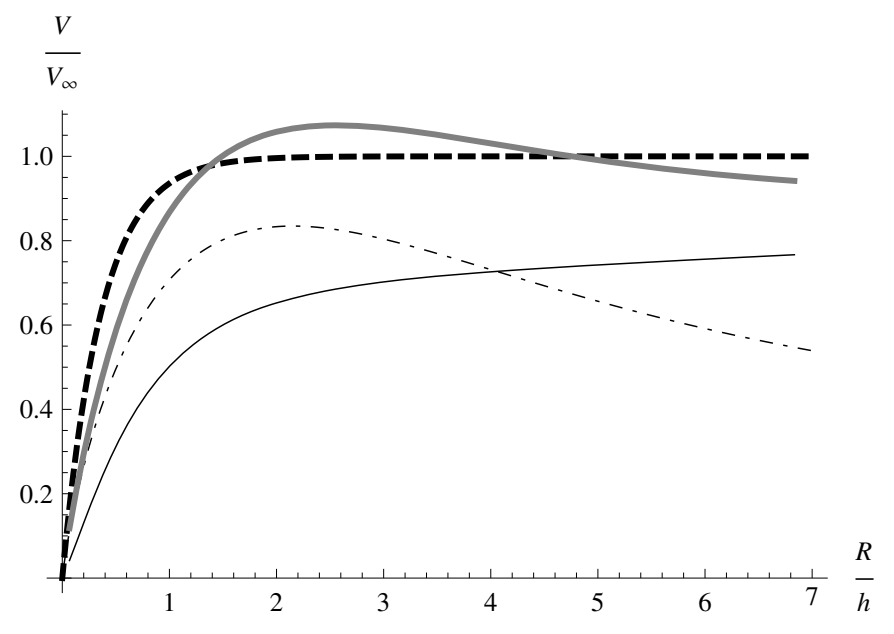

Fig. B.3. Disk-halo decomposition associated with the best selfconsistent fit of the case $\tau_{\mathrm{f}}=0.4$. The coding is the same as in Fig. 12 .

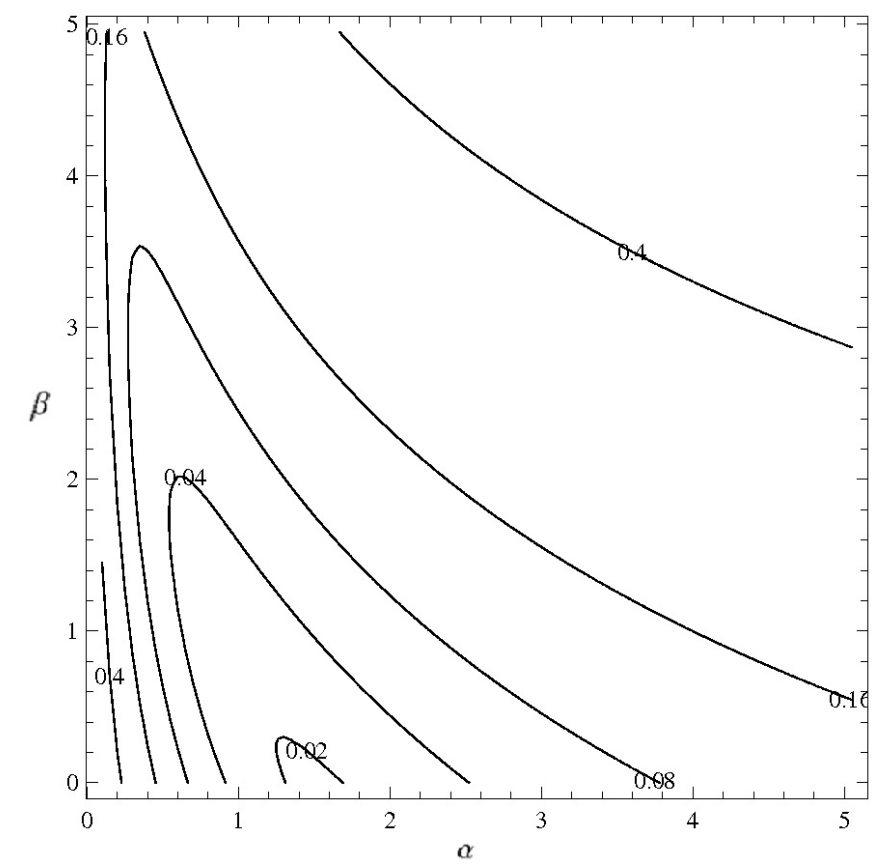

Fig. B.4. Contours of the function $\Xi$ for the parametric decomposition (based on Eqs. (1), (2)) of the rotation curve defined by Eq. (25) with $\tau_{s}=2.3$.

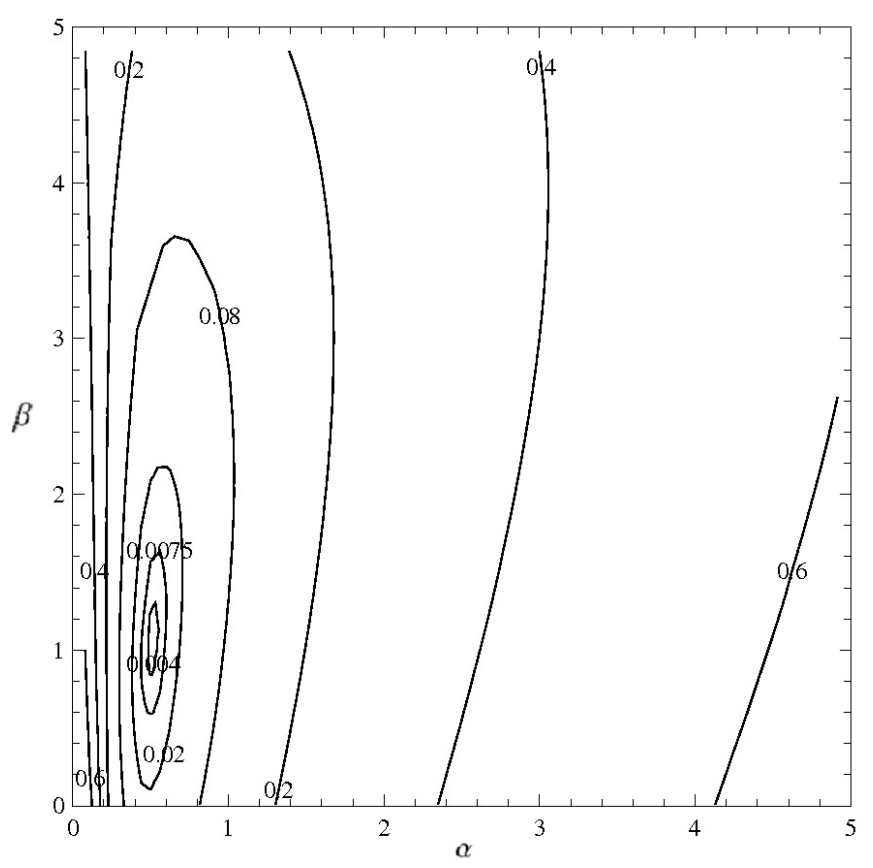

Fig. B.5. Contours of the function $\Xi$ for the self-consistent decomposition of the rotation curve defined by Eq. (25) with $\tau_{\mathrm{s}}=2.3$.

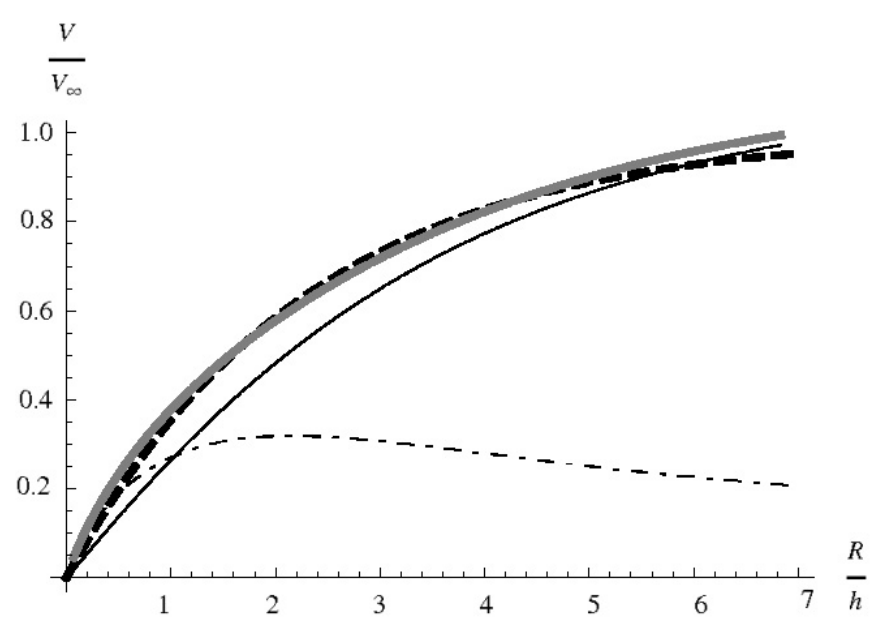

Fig. B.6. Disk-halo decomposition associated with the best selfconsistent fit of the case $\tau_{\mathrm{s}}=2.3$. The coding is the same as in Fig. 12 . 